\title{
Indigo chromophores and pigments: structure and dynamics
}

\author{
V. V. Volkov, ${ }^{a}$ R. Chelli, ${ }^{b}$ R. Righini, ${ }^{c}$ C. C. Perry ${ }^{a}{ }^{*}$ \\ ${ }^{a}$ Interdisciplinary Biomedical Research Centre, School of Science and Technology, Nottingham Trent University, Clifton Lane, Nottingham \\ NG11 8NS, United Kingdom \\ ${ }^{b}$ Dipartimento di Chimica, Università di Firenze, Via della Lastruccia 3, Sesto Fiorentino, I-50019 Italy \\ European Laboratory for Nonlinear Spectroscopy (LENS), Università di Firenze, Via Nello Carrara 1, Sesto Fiorentino, I-50019 Italy
}

\begin{abstract}
In this study, we explore the molecular mechanisms of the stability of indigo chromophores and pigments. Assisted with density functional theory, we compare visible, infrared and Raman spectral properties of model molecules, chromophores and pigments derived from living organisms. Using indigo carmine as a representative model system, we characterize the structure and dynamics of the chromophore in the first electronic excited state using femtosecond visible pump-infrared probe spectroscopy. Results of experiments and theoretical studies indicate that, while the trans geometry is strongly dominant in the electronic ground state, upon photoexcitation, in the Franck-Condon region, some molecules may experience isomerization and proton transfer dynamics. If this happens, however, the normal modes of the trans geometry of the electronic excited state are reconfirmed within several hundred femtoseconds. Supported by quantum theory, first, we ascribe stabilization of the trans geometry in the Franck-Condon region to the reactive character of the potential energy surface for the indigo chromophore when under the cis geometry in the electronic excited state. Second, we suggest that a conical intersection crossing, due to the high barrier along the isomerization pathway in the ground state, would provide for the effective relaxation and observed dominance of the trans geometry of the chromophore in the ground state. Planarity of the chromophore under the trans geometry assists effective dissipation of energy via a cascade of in-plane C-C, C-O $\cdots H-N$ stretchings and C-C-C bending modes delocalized over the molecular mainframe. The described mechanisms help to explain the remarkable photo-stability of indigo chromophores.
\end{abstract}

Keywords: Indigo, Raman, Infrared, Density Functional Theory, Excited State

\footnotetext{
* Corresponding author. Tel.: +0-000-000-0000 ; fax: +0-000-000-0000 ; e-mail: author@institute.xxx .
} 


\section{Introduction}

Indigo pigments can be extracted from many different sources. There are several plants, including Isatis tinctoria, and mollusc shells which accumulate chemical precursors suitable for pigment formation. All indigo dyes include a central double bond (connecting the two heterocyclic indole ring systems), together with the heterocyclic nitrogen atoms and carbonyl groups [1,2], see Fig. 1. For the extent of electronic conjugation, indigo demonstrates a significant bathochromic shift of the energy gap between the highest occupied molecular orbital (HOMO) and the lowest unoccupied molecular orbital (LUMO). This provides the blue-purple coloration for these molecules when in a polar solvent [3-7].

Indigo and its derivatives are very ancient dyes. Extraction of indigo pigments is a signature of Mediterranean/Middle Eastern civilizations. Archaeology reports on mollusc pigment extraction sites, dated between 1800 and 1600 B.C.E., around the Mediterranean and Persian Gulf [8-11]. Royal purple dyed textiles were found in a Bronze Age royal tomb at Qatna in Syria [12]. The book of Exodus commemorates tekhelet pigment to colour cotton fibres, see images of fibres from Ptil Tekhelet in Fig. 1. Usage of these pigments in Western civilization has its own history. The first record of the dye under its Latin name "indigo", as a product of India, was by Pliny [13]. The Roman Empire sustained a set of sumptuary laws, which restricted the use of indigo (among purple and carmine colorants) to the top governing class. The decline of the empire moved the indigo-based color traditions [8-9] into oblivion. It was only when Vasco da Gama established a route to India in 1498, Portuguese Estado da India reintroduced the colorant to Western Europe exporting the material from the Coromandel Coast and Surat. Later, provided by the British East India Company, the annual export of Bengal indigo reached its maximum in 1895: 9,366 tons valued at $3,566,700$ British pounds [14]. However, in 1869, Adolf von Baeyer reported on synthesis of the molecule [15], and the placement of synthetic indigo on the market in 1897 lead the export from India to immediately collapse [14].

What makes this group of colorants so special? Indigo pigments demonstrate a great variance in chemistry of the side groups at different ring positions [1,2]. When present in the solid form, of course, the color of pigments is also affected by intermolecular Hydrogen bonds and exciton coupling. As a result, indigo based materials demonstrate remarkable variability in color in the visible and ultraviolet (UV) spectral regions. For example, in Fig. 2 we demonstrate experimentally detected (DMSO suspensions/solutions) and theoretically predicted (isolated model molecules) variances of the optical absorption from indigo dyes of different sources. Besides the obvious and unique colorations, indigo derivatives attract attention for their remarkable stability and nonhazardous nature: for example, indigo carmine known as E132 or Food Blue 1 is an accepted food additive.

Initially, stability of indigo chromophores was attributed to intra-molecular Hydrogen bonding between the two adjacent carbonyl and $\mathrm{NH}$ groups which lock the molecule in a trans planar configuration, thus preventing the photochemical trans-cis
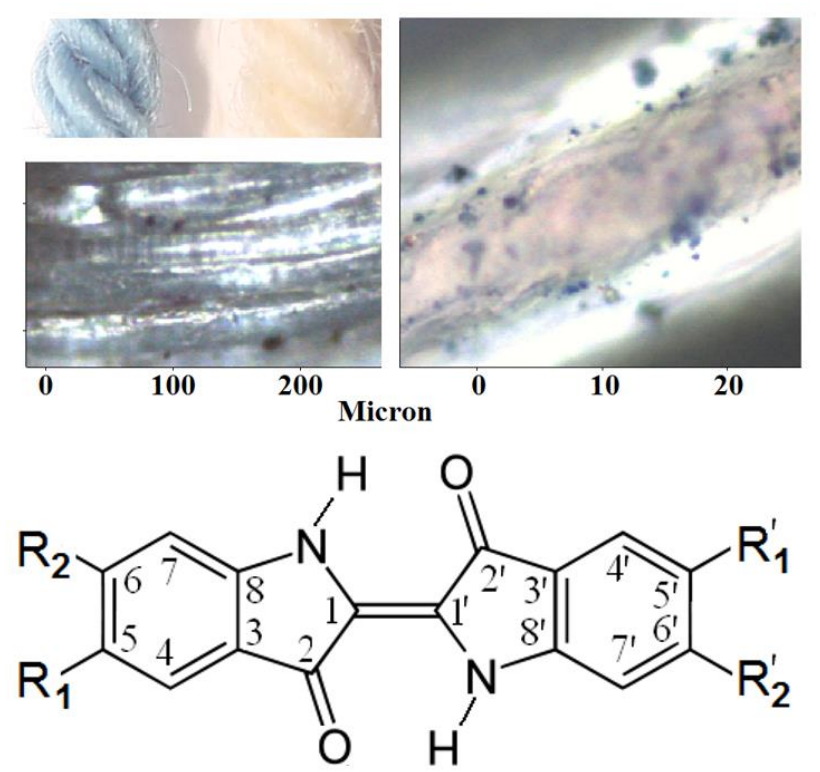

Fig. 1. Images of cotton fibres coloured with polycrystalline inclusions of indigo based tekhelet pigments extracted from Hexaplex trunculus, Ptil Tekhelet. Chemical structure of indigo pigments where $\mathrm{R}$ present a functional group: see main text.

isomerization [16]. In the case of solid pigments, intermolecular interactions were considered to play a stabilizing role, also $[17,18]$. Early time-resolved studies of indigo chromophores in solution indicated that $S_{1}$ deactivation processes occur mainly by internal conversion from the first electronic excited state to the ground state rather than via fluorescence or intersystem crossing or photochemistry [19]: furthermore, Kobayashi and Rentzepis suggested that the apparent internal conversion is associated with fast intra- or/and inter-molecular proton transfer leading to anticipated increases ( 106 times) of the acidity of aromatic $\mathrm{NH}$ and basicity of carbonyls in the first excited electronic state. Indeed, the fluorescence, with lifetimes ranging from 110 to 323 picoseconds, was reported to be of very low quantum yield: $0.0014 \pm 0.0005$ for tetra-methyl-indigo [20], 0.0015 for indigo carmine [21] and 0.0019 for indigo [22]. However, the results of the first visible pump-infrared probe time-resolved studies [20] did not confirm the role of proton transfer in relaxation. Recent Fourier transform Raman analysis of time-dependent modulations of the ultrafast electronic response of indigo carmine in the visible suggests that one of the protons in the $\mathrm{NH} \cdots \mathrm{O}=\mathrm{C}$ pairs may experience intermediate dynamics between nitrogen and oxygen atoms for about $100 \mathrm{fs}$ with apparently, a subsequent recovery of the initial geometry [23].

The main goal of this study is to explore the molecular mechanisms of stability of indigo chromophores. Considering the remarkable diversity in chemistry of the side groups for these chromophores, and that their influence on electronic properties and colour is significant, in this study we combine experimental and theoretical tools to characterize and compare the electronic and vibrational properties of indigo chromophores of various sources. Specifically, first, using UV-VIS, Raman and FTIR we characterize chromophores from plants, molluscs and the products of industrial synthesis extracted into DMSO suspensions or solutions. Next, we discuss 


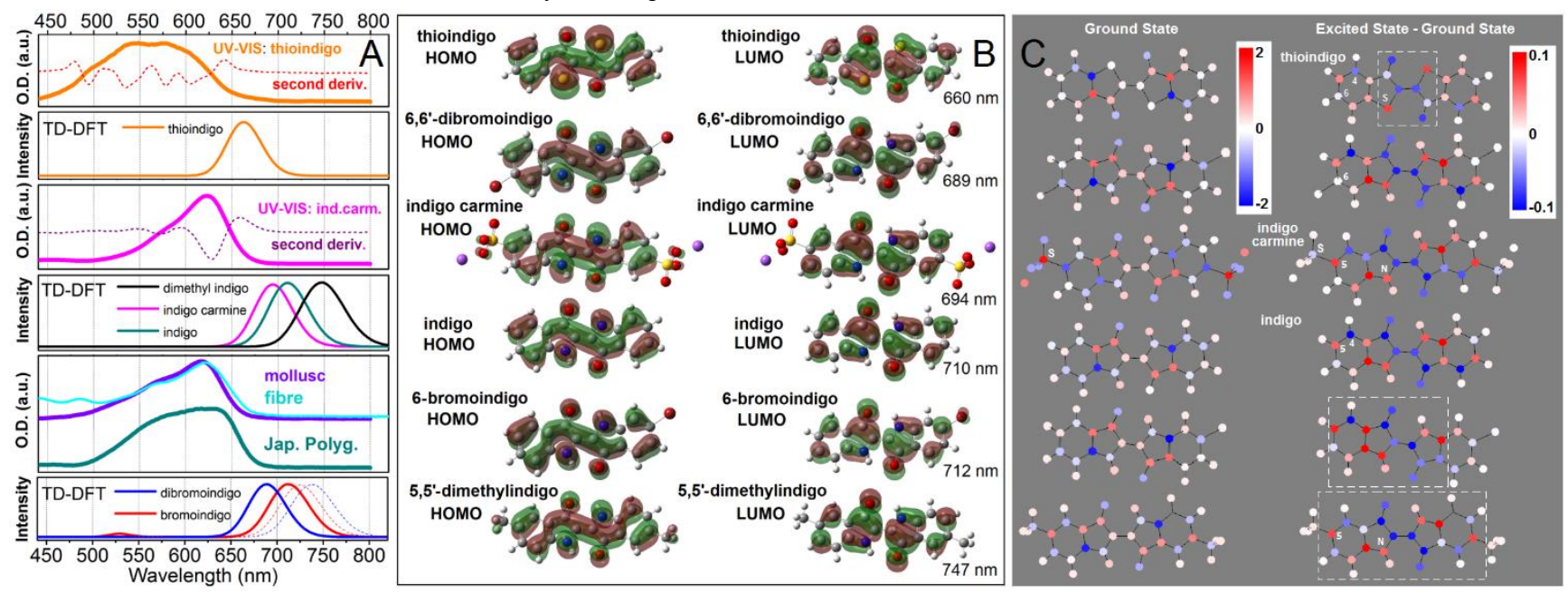

Fig. 2. Panel A: UV-VIS Optical Absorption spectra (thick lines) of indigo chromophores measured in DMSO suspensions/solutions and theory predicted spectra (thin lines) for trans conformers of isolated model molecules: indigo (dark cyan), 5,5'-dimethylindigo (black), indigo carmine, $\mathrm{C}_{16} \mathrm{H}_{8} \mathrm{~N}_{2} \mathrm{Na}_{2} \mathrm{O}_{8} \mathrm{~S}_{2}$ (magenta), and thioindigo, $\mathrm{C}_{16} \mathrm{H}_{8} \mathrm{O}_{2} \mathrm{~S}_{2}$ (orange). Blue and red lines show calculated results for 6-bromoindigo, 6,6'-dibromoindigo, respectively. Thin blue and red dashed lines show calculated results for 5-bromoindigo, 5,5'-dibromoindigo, respectively. The spectra were calculated taking convolutions with Gaussian line-shape with the width according to the fit of experimental spectrum of indigo carmine as provided in Supporting Information file. Panel B: chemical structures of the indigo isolated model molecules (except 5-bromoindigo, 5,5'-dibromoindigo) together with the molecular orbitals involved in optical excitation in the visible spectral range. Panel C: left column of structural images shows the colour maps of the Mulliken charges of the atoms of the model molecules in their electronic ground state; right column of structural images shows the colour maps of the changes of Mulliken charges of the atoms upon transition from the electronic ground state to the first electronic excited state. The variances of dashed line boxes help to see the effect of $2 \mathrm{D}$ structural confinement to explain variances of the visible spectra as discussed in the text.

the experimental results comparatively with assistance of quantum chemistry predictions. On one hand, this helps to relate our results to properties of various indigo pigments of natural origin. On the other hand, the approach guides us from a general account of electronic and structural properties of such chromophores to specific research on structural dynamics for a representative case, indigo carmine in DMSO solution, using properly specified vibration markers. Practically, we achieve this by conducting femtosecond Visible pump - IR probe experiments, and exploring Fourier Transform of low frequency Raman active modulations of time-resolved IR signatures specific to the structure in the first electronic excited state. As a result, we discuss the relaxation mechanisms which may explain the remarkable stability of indigo chromophores and related solid pigments.

\section{Experimental}

\subsection{Pigments and chemical components}

Cotton fibres coloured with indigo pigment (tekhelet) and crushed hypobranchial glands from Murex trunculus were obtained from Ptil Tekhelet, Jerusalem. Thioindigo, $\mathrm{C}_{16} \mathrm{H}_{8} \mathrm{O}_{2} \mathrm{~S}_{2}$, genuine indigo solid extract from Indigofera sp. $\left(\mathrm{C}_{16} \mathrm{H}_{10} \mathrm{~N}_{2} \mathrm{O}_{2}\right.$ with impurities), indigo solid extract from Polygonum tinctorum and tyrian purple (brominated indigo pigments with impurities) were obtained from Kremer Pigmente GmbH \& Co. KG, Aichstetten, Germany. Indigo carmine, $\mathrm{C}_{16} \mathrm{H}_{8} \mathrm{~N}_{2} \mathrm{Na}_{2} \mathrm{O}_{8} \mathrm{~S}_{2}$, was obtained from Sigma-Aldrich, CAS Number: 860-22-0. Dimethyl sulfoxide (DMSO) from Aldrich CAS 67-68-5 was used for resonant Raman and FTIR measurements. In the case of indigo carmine we used deuterated DMSO, Aldrich CAS 2206-27-1 to gain better detection in the spectral range from 1000 to $1800 \mathrm{~cm}^{-1}$.

\subsection{Steady state spectroscopy}

FTIR and ATR-FTIR studies were conducted using a Nicolet 6700 FTIR spectrometer and an Attenuated Total Reflection (ATR) unit Model: 0055290 from Thermo Electron, now, Thermo Fisher Scientific. Raman spectra were measured using a DXR microscope station from Thermo Fisher Scientific, Madison, Wisconsin, equipped with 50x and 100x microscopy objectives. The spectral resolution in the Raman experiment was down to 2 $\mathrm{cm}^{-1}$ according to the instrumental limit of the microscope operated with a 25 micron confocal slit. Raman studies were conducted using samples deposited on an unprotected gold mirror, PFSQ05-03-M03 Thorlabs Ltd., Ely, United Kingdom. Resonant Raman measurements were made using $530 \mathrm{~nm}$ excitation radiation of 1-5 $\mathrm{mW}$ on chromophores in droplets of suspensions in DMSO under 300 micron thick cover glass slips. Non-resonant Raman measurements were conducted on solid samples (pigments) using radiation at $780 \mathrm{~nm}$ of $10 \mathrm{~mW}$.

\subsection{Time-resolved spectroscopy}

Time-resolved responses were detected using a spectrometer (European Laboratory for Nonlinear Spectroscopy, Sesto Fiorentino) based on a Micra-Legend Elite laser-amplifier system from Coherent Inc., Santa Clara, USA. The system produces a 1 $\mathrm{kHz}$ train of $35 \mathrm{fs}$ (FWHM), $3 \mathrm{~mJ}$ pulses at $800 \mathrm{~nm}$. The output was split to pump an optical parametric amplifier (OPA) TOPAS (Light Conversion Ltd., Vilnius, Lithuania) and a home-built OPA [24].

To conduct Visible pump/IR probe experiments, first, we prepared Visible pump pulsed radiation for $\mathrm{S}_{0}-\mathrm{S}_{1}$ electronic excitation, between the electronic ground $\left(\mathrm{S}_{0}\right)$ and the lowest electronic excited states $\left(\mathrm{S}_{1}\right)$ of indigo carmine in DMSO solution. 
Specifically, for this task, we used 100 fs pulses of $50 \mu \mathrm{J}$ with the wavelength of the spectral envelope centred at $626 \mathrm{~nm}$, and with the spectral width of the visible radiation at $360 \mathrm{~cm}^{-1}$. Such pulses were prepared by doubling the frequency of the signal radiation from the TOPAS with a second harmonic crystal. Furthermore, we included in the path two prisms (as a spectral filter) to eliminate residual signal, idler and other radiations. This helped ensure that the excitation radiation would contain the spectral components centred at $626 \mathrm{~nm}$, only. For the probe pulse we used pulsed midIR radiation, generated by means of the home-built OPA [24]. The energy was $2 \mu \mathrm{J}$. The central frequency was tunable between 1000 and $3000 \mathrm{~cm}^{-1}$. The spectral width of the mid-IR radiation was about $200 \mathrm{~cm}^{-1}$. Before reaching the sample, a fraction of the probe pulse was split off and used as a reference. After the sample, both probe and reference pulses are spectrally dispersed in a spectrometer TRIAX 180, HORIBA Jobin Yvon, Milano, and imaged separately on a 32 channel double array Mercury Cadmium Telluride detector from InfraRed Associates Inc., Florida, USA. The ratio of the probe to the reference spectra gave the Visible pump/IR probe spectrum which was recorded as a function of the pump-probe time delay.

\subsection{Closed cycle flow-cell system}

To perform time-resolved measurements we used a homemade closed cycle flow-cell system, where a FMI LAB Pump (Model QD2/QD2B) with RH0CKC head from Fluid Metering Inc. was used to circulate the sample. The design of the flow-cell, in general, resembles that reported in Ref. 25. The main difference is that we avoided using O-rings in the contacts of $0.5 \times 1.5 \mathrm{~mm}$ polytetrafluoroethylene tubes (C31140, Novodirect $\mathrm{GmbH}$, Germany) with $\mathrm{CaF}_{2}$ windows of the cell. Sealed contacts were achieved by a proper pressing of the funnel terminations of the tubes directly to the windows by designing the flow-cell with thicker walls to accommodate threaded connectors from Omnifit Ltd. With this design it was possible to have a controllable flow rate in the range of 0 (no flow) $-1 \times 10^{-7} \mathrm{~m}^{3} / \mathrm{sec}$. Considering the 1 $\mathrm{KHz}$ repetition rate, the thickness of the spacer of $100.10^{-6} \mathrm{~m}$, the width of the flow channel of $2.10^{-3} \mathrm{~m}$ and that the beam diameter could be focused to be smaller than $70.10^{-6} \mathrm{~m}$, it was possible to refresh the sample for every incoming excitation pulse.

\subsection{Density functional theory}

The quantum chemical calculations of the vibrational spectra of isolated indigo carmine in the electronic ground $\left(\mathrm{S}_{0}\right)$ and the first electronic excited states $\left(S_{1}\right)$ were performed using the 6$31++\mathrm{g}(\mathrm{d}, \mathrm{p})$ basis set and the restricted B3LYP functional [26,27] within the Gaussian 09 program package [28]. DMSO may form a Hydrogen bond with $\mathrm{NH}$ moieties of indigo. To account for this implicitly, we adopt a polarizable continuum model [29]. In the Supporting Information file we provide results of DFT studies the article, when the role of solvent is accounted for using polarizable continuum model (PCM). At the same time, the energy of electronic transitions for indigo carmine with two DMSO molecules is closer to that observed in experiment.

To address the vibrational and electronic properties of indigo chromophores we conducted DFT and Time-Dependent DFT (TD-DFT) calculations on trans conformers of isolated model molecules: indigo $\left(\mathrm{R}_{1}=\mathrm{R}_{2}=\mathrm{R}^{\prime}{ }_{1}=\mathrm{R}^{\prime}{ }_{2}=\mathrm{H}\right)$, 6-bromoindigo $\left(\mathrm{R}_{1}=\mathrm{R}^{\prime}{ }_{1}=\mathrm{R}_{2}=\mathrm{H}, \quad \mathrm{R}^{\prime}{ }_{2}=\mathrm{Br}\right), \quad 6,6^{\prime}$-dibromoindigo $\quad\left(\mathrm{R}_{1}=\mathrm{R}^{\prime}{ }_{1}=\mathrm{H}\right.$, $\left.\mathrm{R}_{2}=\mathrm{R}^{\prime}{ }_{2}=\mathrm{Br}\right), \quad 5,5^{\prime}$-dimethylindigo $\quad\left(\mathrm{R}_{2}=\mathrm{R}^{\prime}{ }_{2}=\mathrm{H}, \quad \mathrm{R}_{1}=\mathrm{R}^{\prime}{ }_{1}=\mathrm{CH}_{3}\right)$, indigo carmine $\left(\mathrm{R}_{2}=\mathrm{R}^{\prime}{ }_{2}=\mathrm{H}, \mathrm{R}_{1}=\mathrm{R}^{\prime}{ }_{1}=\mathrm{SO}_{3}^{-} \ldots \mathrm{Na}+\right)$, and thioindigo, see Fig. 1 and 2. To contrast the effect of residue position on electronic properties, we calculate UV-VIS spectra for 5bromoindigo $\left(\mathrm{R}_{1}=\mathrm{Br}, \mathrm{R}^{\prime}{ }_{1}=\mathrm{R}_{2}=\mathrm{R}^{\prime}{ }_{2}=\mathrm{H}\right)$ and for 5,5'-dibromoindigo $\left(\mathrm{R}_{1}=\mathrm{R}^{\prime}{ }_{1}=\mathrm{Br}, \mathrm{R}_{2}=\mathrm{R}^{\prime}{ }_{2}=\mathrm{H}\right)$. To describe possible structural variances of indigo carmine in the electronic excited state, additionally, we perform calculations for the cis conformer, and for a $30^{\circ}$ twist conformer of this molecule, where the planes of the two heterocyclic indole ring systems depart from the parallel geometry (as in the trans conformation) by $30^{\circ}$. Here, it is necessary to notice that DFT and TD-DFT optimization of indigo carmine under $c$ is geometry in the ground and in the first electronic excited state show the planes of the two heterocyclic indoles to be twisted at the $\mathrm{C}_{2}-\mathrm{C}_{1}-\mathrm{C}_{1^{\prime}}-\mathrm{C}_{2^{\prime}}$ angle by about $11^{\circ}$ and $35^{\circ}$, respectively. This is because of the weak repulsion between facing N-H moieties and $\mathrm{C}=\mathrm{O}$ moieties. Energetics of proton transfer for indigo carmine in the electronic excited state were explored considering that transfer would happen only on one side of the molecule. Transfer of the second proton on the other side of the molecule as well as a concerted pathway, which allows both protons to be transferred at the same time, were not considered based on the results of previous studies [23].

To plot IR and Raman spectra in different spectral regions we adopted three factors to scale the calculated frequencies. Specifically, below $300 \mathrm{~cm}^{-1}$ the scaling factor was 1 ; in the midIR spectral range, between 1000 and $2000 \mathrm{~cm}^{-1}$, the scaling factor was 0.97 ; for the frequency range, above $3000 \mathrm{~cm}^{-1}$, the scaling factor of 0.91 was used.

\section{Results and discussion}

\subsection{UV-VIS optical properties}

In Fig. 2A we present a series of steady-state UV-VIS optical absorption spectra of: i) DMSO extract (in the form of a suspension) of indigo pigments from: Ptil Tekhelet, ii) indigo carmine, iii) DMSO suspension from a solid pigment condensation for Indigofera $s p$. and iv) thioindigo. First, the response of indigo carmine in the visible spectral range demonstrates an optical absorption, which we may fit with two broad Gaussians: one to match the electronic transition at $628 \mathrm{~nm}$, and another to fit the contribution of the vibrational progression, which is centred at about $530 \mathrm{~nm}$. The data suggest a FranckCondon vibronic envelope: the fitting is provided in the Supporting Information file. The observed optical absorption is specific to the $\pi-\pi^{*}$ transition and characteristic for such chromophores [2,4-7,16,21].

Next, the response of a DMSO suspension of pigments from the hypobranchial glands of Murex trunculus demonstrates a

Table I. Calculated electronic and structural properties of isolated model molecules in the ground state. Numbers in brackets show the changes of the corresponding bond lengths when the molecules are in the first electronic excited state. In the case of asymmetric 6-bromoindigo, the first numbers are for the moieties on the side of the molecule where the Bromine atom is located. 
Dyes and Pigments xxx (2019) xxx-xxx

\begin{tabular}{|l|l|l|l|l|l|}
\hline $\mathrm{R}$ group & $\lambda_{0} / \mathrm{nm}$ & $\mathrm{C}=\mathrm{O} / \AA$ & $\mathrm{C}=\mathrm{C} / \AA$ & $\mathrm{C}=\mathrm{O}-\mathrm{H}_{\mathrm{N}}(\mathrm{S}) / \AA$ & $\Sigma_{\mathrm{i}} \mid \delta_{i} \mathrm{r}_{\mathrm{i}} / \mathrm{au} \AA$ \\
\hline $\mathrm{CH}_{3}$ & 747.1 & $1.241(+1.37 \%)$ & $1.366(+1.79 \%)$ & $2.299(-0.22 \%)$ & 4.28 \\
\hline $1 \mathrm{Br}$ & 712.0 & $1.239(+1.44 \%)$ & & $2.294(+2.5 \%)$ & 4.69 \\
& & $1.240(+1.30 \%)$ & $1.365(+1.82 \%)$ & $2.304(-3.3 \%)$ & \\
\hline $\mathrm{H}$ & 710.3 & $1.240(+1.35 \%)$ & $1.366(+1.89 \%)$ & $2.300(-0.4 \%)$ & 4.32 \\
\hline $\mathrm{SO}{ }_{3} \mathrm{Na}$ & 694.2 & $1.238(+1.33 \%)$ & $1.364(+1.97 \%)$ & $2.303(-0.546 \%)$ & 4.61 \\
\hline $2 \mathrm{Br}$ & 688.7 & $1.239(+1.30 \%)$ & $1.364(+1.98 \%)$ & $2.300(-0.6 \%)$ & 3.96 \\
\hline $\mathrm{S}$ & 660.1 & $1.232(+1.52 \%)$ & $1.360(+2.26 \%)$ & $2.831(+1.9 \%)$ & 2.69 \\
\hline
\end{tabular}

slightly broader (if we compare to that of indigo carmine) band with the main feature centred at about $617 \mathrm{~nm}$ and broadening on the blue side, mainly. The optical absorption of the DMSO suspension from a mineralized condensate of plant origin (Indigofera sp., Kremer Pigmente $\mathrm{GmbH}$ ) is rather complex. It contains an apparent spectral signature at $640 \mathrm{~nm}$ and a very broad spectral dispersion (down to $500 \mathrm{~nm}$ ) on the blue side. In the series of spectra being compared, the response of thioindigo is the most blue-shifted, compared to the other experimentally detected responses.

To understand the variances in the measured UV-VIS spectral responses, we adopt TD-DFT to anticipate electronic and structural properties of the model molecular systems, as listed in the Materials and methods: see Fig. 2 and Table 1, respectively. The data presented in Fig. 2B show systematic behaviour and yet do not help to explain the spectral variances. Also, overall, the structural properties of the chromophores listed in Table 1 in relation to predicted wavelengths of HOMO-LUMO transitions do not suggest any obvious correlation to rationalize the observed electronic properties. We may notice, however, that there is a subtle wavelength dependence on the percentage of the change of the length of the bond which connects the two heterocyclic indole rings which sorts thioindigo and dimethyl indigo at the opposite ends of the list.

Since indigo molecules are systems where $\pi$ electrons may conjugate not only along the long axis, to understand better what happens upon the transition to the first excited state, in Fig. 2C, we present colour maps of the Mulliken atomic charges (when in the ground states) and difference Mulliken charges (charges in the excited state minus charges in the ground state) structurally mapped to the positions of the atoms in two-dimensional space of the molecular frames. The charge difference maps are helpful in visualizations of predicted complexity of electronic delocalization in such molecular systems where two-dimensional electronic spatial correlation effects may become compromised upon three dimensional distortions.

Alterations of atomic charges upon electronic excitations were considered before [30], but not as a tool to address the effects of structural confinement on electronic correlations to explain optical properties. Specifically, examining the Mulliken charge difference maps one may notice the relatively blue shifted character of UVVIS absorption for thioindigo [31,32] - as the main electronic differences are rather localized on the $\mathrm{CO}$ and $\mathrm{S}$ atomic groups, within the 2D structural confinement: see the dash-line box in Figure 2C. In contrast, dimethyl-indigo, with its electronic increment at carbon atoms at positions 1, 2, 3 and 4, demonstrate the lowest energy electronic resonances. The situation is similar in indigo carmine save the sulfur atoms are electron withdrawing. The situation with 6-bromoindigo and 6,6'-dibromoindigo is more intriguing. In the case of 6-bromoindigo, the strong and rapid electron difference on atoms 3, 4 and 5 (while the Bromine atom is next to $6^{\prime}$ ) is compensated by differential delocalization mainly on one half of the molecule. In the case of 6,6'-dibromoindigo, the strong electron variance associated with the HOMO-LUMO transition is strongly localized on the two heterocyclic structural elements. This relative $2 \mathrm{D}$ confinement may explain the relative blue shift of the electronic response for this molecule. This becomes obvious if we compare sums for $\left|\delta_{i} r_{i}\right|$ values for the considered molecule, where $\delta_{i}$ and $r_{i}$ are the partial atomic charge and the distance from the centre of $\mathrm{C}_{1}-\mathrm{C}_{1}$, bond for an atom $\mathrm{i}$. The role of radial value $r_{i}$ instead of any distance, (as shown in Table 1) is helpful to sense that the nature of the electronic resonance in the indigo system reflects the physics of $2 \mathrm{D}$ confinement. Here, it is interesting to note that calculated for this study, the UV-VIS optical spectra for 5-bromoindigo and 5,5'-dibromoindigo anticipate the opposite - a bathochromic shift for the lowest electronic transition, see the dashed red and blue lines in in Fig. 2A. Finally, and this is important for our discussion later; upon excitation to the first electronic excited state, all indigo systems considered here demonstrate strengthening in electric interactions between the $\mathrm{C}=\mathrm{O}$ and the $\mathrm{H}-\mathrm{N}$ moieties or the sulfur atoms.

With the provided theoretical visual perspective, we may return to interpretation of the experimental UV-VIS optical absorption spectra, as shown in Fig. 2A. In respect to the spectral response detected from the DMSO suspension of pigments from the hypobranchial glands of Murex trunculus, here, it is also helpful to note that previous studies on similar compounds revealed that the source may contain indigo, indirubin, 6bromoindigo and 6,6'-dibromoindigo [7,12,33], and the contributions of such molecules would depend on mollusc species [7], solvent [7], and the character of material processing [7,12,33]. As we have mentioned, our DMSO extract (in the form of a suspension) of the pigments from Murex trunculus demonstrates a slight blue side broadened band (comparing to that of indigo carmine) with the main feature centred at about $617 \mathrm{~nm}$. Accordingly, we may ascribe the spectral main feature and the slight enrichment of the spectral response on the blue side to the presence of 6,6'-dibromoindigo among other possible chromophores and to a lesser presence of indirubin and 6'monobromoindirubin. Here, we suggest that the spectral response of the DMSO extract (in the form of a suspension) of pigments from a mineralized condensate of plant origin (Indigofera $s p$., Kremer Pigmente $\mathrm{GmbH}$ ), may be explained by the spectral contribution of indigo at about $640 \mathrm{~nm}$ (consistently, the calculated resonance of indigo is red shifted compared to that of indigo carmine), and a significant presence of indirubin molecules contributing to optical density at $550 \mathrm{~nm}[7,12,33]$.

\subsection{Raman and infrared responses in the electronic ground state}

To tackle the photo-stability of indigo chromophores we 

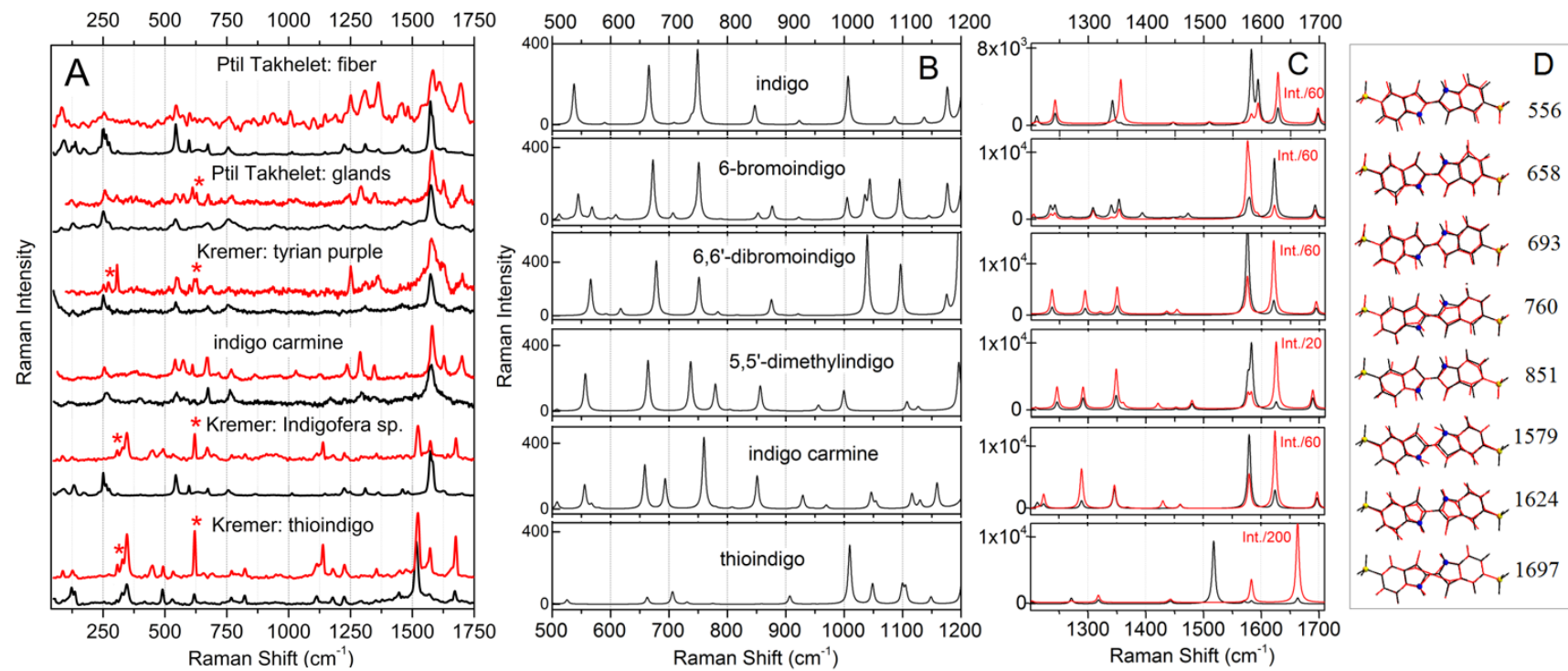

Fig. 3. Panel A: non-resonant (black lines) and resonant (red lines) Raman spectra for pigment and chromophore containing samples, as indicated. Stars mark resonances where solvent may contribute strongly. Panels B and C: Raman spectra calculated for model molecules: indigo, 6-bromoindigo, 6,6'dibromoindigo, 5,5'-dimethylindigo, indigo carmine and thioindigo; red lines in $\mathrm{C}$ show calculated pre-resonant Raman spectra. The scaling factor for the calculated frequencies is 0.97. Panel D: Graphical representation of the main Raman active normal modes of indigo carmine by DFT. The Supporting Information file provides larger graphical views of the atomic displacements for these modes.

should probe its structure and dynamics in the electronic excited state. Such research requires, first, learning on the normal modes of the molecules when in the ground state and how they reflect structural properties. Second, we have to anticipate the corresponding normal modes and their frequencies when an indigo molecule is in the first electronic excited state. In developing our approach, first we explore Raman and infrared responses, experimentally and theoretically, for the considered chromophores when in the electronic ground state.

In Fig. 3, we present experimentally detected Raman (Panel A) for the pigment extracts and calculated Raman (Panels B and C) responses of the model molecules in the spectral region from 500 to $1700 \mathrm{~cm}^{-1}$. In this spectral range, we may sort the normal modes into three groups where i) C-C-C bendings admix with $\mathrm{C}-\mathrm{C}$ stretchings to provide the delocalized in-plane relative deformations involving all four rings $\left(500\right.$ and $\left.800 \mathrm{~cm}^{-1}\right)$; ii) inplane $\mathrm{C}-\mathrm{C}-\mathrm{H}$ collective bending modes admixed with $\mathrm{C}-\mathrm{C}$ stretchings dominate in the spectral region between 1000 and 1300 $\mathrm{cm}^{-1}$, iii) $\mathrm{C}-\mathrm{C}$ stretchings get admixed with $\mathrm{C}=\mathrm{O}$ stretchings to appear between 1500 and $1700 \mathrm{~cm}^{-1}$. In Fig. 3D, we provide graphical representations of the most intense Raman active normal modes of indigo carmine that dominate in the indicated. spectral region and their frequencies in wavenumbers scaled by 0.97

In the spectral region of $1200-1750 \mathrm{~cm}^{-1}$, we see that nonresonant Raman spectra of all samples (black lines in Fig. 3A), save that of thioindigo, demonstrate similar responses. It is interesting that we observe reasonable agreement between nonresonant and resonant Raman responses from pigmented cotton fibre (Ptil Takhelet), indigo carmine, the hypobranchial glands of Murex trunculus (Ptil Tekhele), tyrian purple mollusc extract (Kremer Pigmente) and from thioindigo. It is worth noting that while the resonant Raman signatures of pigments (in suspension) from the crushed hypobranchial glands of Murex trunculus (Ptil Tekhelet) resemble that from tyrian purple, 6,6'-dibromoindigo with impurities in solid form (Kremer Pigmente GmbH \& Co), the resonant Raman from tekhelet fibre (as in Fig. 1) demonstrates broader resonances and the highest frequency mode in this manifold is strongly enhanced. This suggests several possible reasons: i) structural selectivity for pigments to partition into cotton fibre, ii) role of site variances when in the fibre matrix; anisotropy in molecular orientation when a Raman signal is detected in reflection geometry.

The resonant Raman response of chromophores extracted from mineralized condensates of plant origin (Indigofera sp., Kremer Pigmente $\mathrm{GmbH}$ ) is significantly different from that under nonresonant excitation. This reflects the role of the Franck-Condon factors. In fact, calculations of pre-resonant Raman for indigo, bromoindigo and indigo carmine (see Fig. 3C) demonstrate good similarity with the spectra of indigo containing samples (see Fig. $3 \mathrm{~A})$ at the 6 micron spectral range.

In the case of thioindigo, the differences in molecular geometry (see Table 1), masses and the absence of the intra-molecular Hydrogen bonds should affect the character of normal modes and of the observed resonances. Let us consider the lower frequency range, $400-1250 \mathrm{~cm}^{-1}$, where normal modes with participation of a sulfur atom may help to explain the observed differences. Using the results of DFT studies, we list here: (i) the state observed at $490 \mathrm{~cm}^{-1}$ (476 $\mathrm{cm}^{-1}$ by DFT) may be due to $\mathrm{C}_{3}-\mathrm{C}_{8}-\mathrm{S} / \mathrm{C}_{3^{\prime}} \mathrm{C}_{8^{\prime}-\mathrm{S}}$ symmetric bending; (ii) the state observed at $823 \mathrm{~cm}^{-1}\left(706 \mathrm{~cm}^{-1}\right.$ by DFT) may represent symmetric compression of benzene rings along $\mathrm{C}_{5} \cdots \mathrm{C}_{8} / \mathrm{C}_{5} \cdots \mathrm{C}_{8}{ }^{\prime}$ directions, and would involve $\mathrm{C}_{8}-\mathrm{S} / \mathrm{C}_{8}-\mathrm{S}$ symmetric stretching; (iii) the Raman peak at $1178 \mathrm{~cm}^{-1}$ (theory anticipates at $1048 \mathrm{~cm}^{-1}$ ) may represent $\mathrm{C}_{8}-\mathrm{C}_{7}-\mathrm{H} / \mathrm{C}_{3}-\mathrm{C}_{4}-\mathrm{H} / \mathrm{C}_{8^{\prime}}-\mathrm{C}_{7^{\prime}}-$

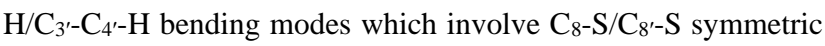
stretching; and (iv) the Raman peak at $1226 \mathrm{~cm}^{-1}$ (DFT predicts to be at $1105 \mathrm{~cm}^{-1}$ ) may involve delocalised in-plane bendings of Hydrogens in the C-C-H moieties with participation of in-phase symmetric $\mathrm{C}_{8}-\mathrm{S}_{-} \mathrm{C}_{1}$ and $\mathrm{C}_{8}{ }^{-}-\mathrm{S}_{-} \mathrm{C}_{1}{ }^{\prime}$ stretchings. The Supporting Information file provides graphical views for the atomic displacements of the normal modes described here. Overall, DFT is able to predict relatively well the 

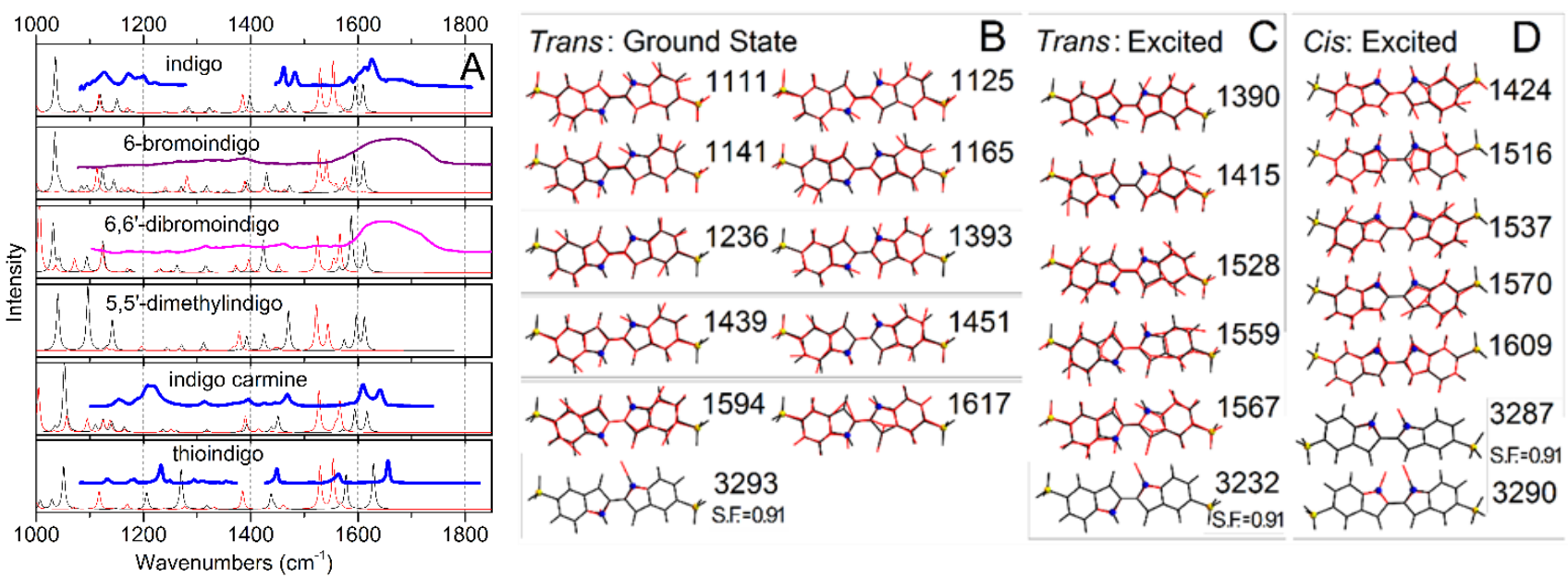

Fig. 4. Panel A: blue lines show experimentally measured FTIR for extracts of chromophores (suspensions in deuterated DMSO) from Japanese polygonum, for a solution of indigo carmine in deuterated DMSO, and for DMSO suspensions of thioindigo; purple and magenta lines show FTIR spectra for pigment extracts (DMSO suspensions) from tyrian purple and crushed hypobranchial glands, respectively; black and red lines show theoretically calculated infrared absorptions of the model molecules, as indicated, in the electronic ground and electronic excited states, respectively. Panels B, C and D: graphical representations of the main infrared active normal modes of indigo carmine model molecule in the ground state trans geometry, in the excited state trans geometry and in the excited state cis geometry, respectively. The scaling factor for the calculated frequencies is 0.97 , save for the NH modes where the factor is 0.91 . Supporting Information file provides larger graphical views of the atomic displacements for these modes.

frequencies of the normal modes in the mid-IR spectral range. Finally, here, it is important to note that the Raman spectra we report are in general agreement with previous Raman studies for indigo carmine [34], bromindigo [35], and indigo [36].

The selectivity rules for IR responses make them complementary to Raman. However, due to the character of contributing transition dipole moments, IR responses could be particular chemical moieties including dependence on environment. Fig. 4A shows representative FTIR spectra (blue lines) for the chromophores (suspension/solution) extracts and IR responses of the model systems in the electronic ground state calculated by DFT (black lines) in the spectral region from 1000 to $1800 \mathrm{~cm}^{-1}$. An intense doublet at 1611 and $1634 \mathrm{~cm}^{-1}$ dominates the experimental response. A comparative review of the spectral features with the results of DFT calculation enables identification of 5 fundamentals of relevant infrared optical intensity for indigo carmine in the mid-IR. The experimentally observed intense resonance at $1634 \mathrm{~cm}^{-1}$ is due to the antisymmetric $\mathrm{C}=\mathrm{O}$ stretching admixed with $\mathrm{C}-\mathrm{C}$ stretching and antisymmetric in-plane bendings of the N-H motifs. The lower frequency transition at $1611 \mathrm{~cm}^{-1}$ is specific to the benzene rings antisymmetric deformations mixed partially with C-H bendings. In the left plate in the right panel we provide the graphical presentations of the DFT predicted atomic displacements for these vibrations at the calculated frequencies of 1617 and $1594 \mathrm{~cm}^{-1}$, respectively. Next, we assign the experimentally observed lower frequency subset of states at 1470 , 1440 , and $1396 \mathrm{~cm}^{-1}$ to in-plane $\mathrm{C}-\mathrm{H}$ and $\mathrm{N}-\mathrm{H}$ bendings. The graphical presentations labelled with scaled frequencies at 1451 , 1439 and $1393 \mathrm{~cm}^{-1}$ demonstrate the atomic displacements, which we consider to be specific to these modes, respectively. The results for indigo carmine are consistent with previous theoretical and experimental studies [6,32,34].

FTIR spectra for pigment extracts (in deuterated DMSO) from tyrian purple and from crushed hypobranchial glands, see Fig. 4, spectra demonstrate intense optical absorption in the spectral region specific to Amide I and Amide II transitions. This arises from a relatively high concentration of proteins in both extracts. The overwhelming Amide absorptions of the DMSO extract (suspension) from tyrian purple restricts molecular assignments. In the case of the DMSO extract (suspension) from glands, the observed infrared signatures at 1317, 1386 and $1461 \mathrm{~cm}^{-1}$ correspond well to calculated resonances at $1316 \mathrm{~cm}^{-1}$ (1317), 1374 (1393) and $1424 \mathrm{~cm}^{-1}\left(1431 \mathrm{~cm}^{-1}\right)$, which are specific to C$\mathrm{C}$ stretchings, C-C-H and $\mathrm{C}-\mathrm{N}-\mathrm{H}$ in-plane bending modes, respectively, as predicted for 6-bromoindigo (6,6'dibromoindigo). Additionally, in this sample, we see the relatively high optical density at the blue side of the band specific to Amide transitions that may be assigned to the contributions of the dominant infrared doublet, which we observe at 1611 and 1634 $\mathrm{cm}^{-1}$ in FTIR of indigo carmine. These observations indicate a relatively higher contribution of genuine indigo in the DMSO extract (suspension) from crushed hypobranchial glands.

In the case of the mineralized condensate of plant origin we observe a rather complex subset of resonances at $1600 \mathrm{~cm}^{-1}$, consistent with the results of Raman and UV-VIS results described above. The FTIR response of thioindigo shows a larger (comparing to such for other chromophores) frequency split for the intense mid-IR doublet at 1565 and $1655 \mathrm{~cm}^{-1}$. DFT studies confirm this tendency with predicted activities at 1580 and 1630 $\mathrm{cm}^{-1}$. The observed and anticipated features reflect both, the significant structural difference of this chromophore from other indigo molecules (Table 1) and the absence of intramolecular hydrogen bonding.

\subsection{Structure and dynamics in the electronic excited state: mid- IR spectral range}

After characterization of the series of indigo chromophores in the electronic ground state, we are ready to address the structural properties and relaxation dynamics of indigo carmine, as a 

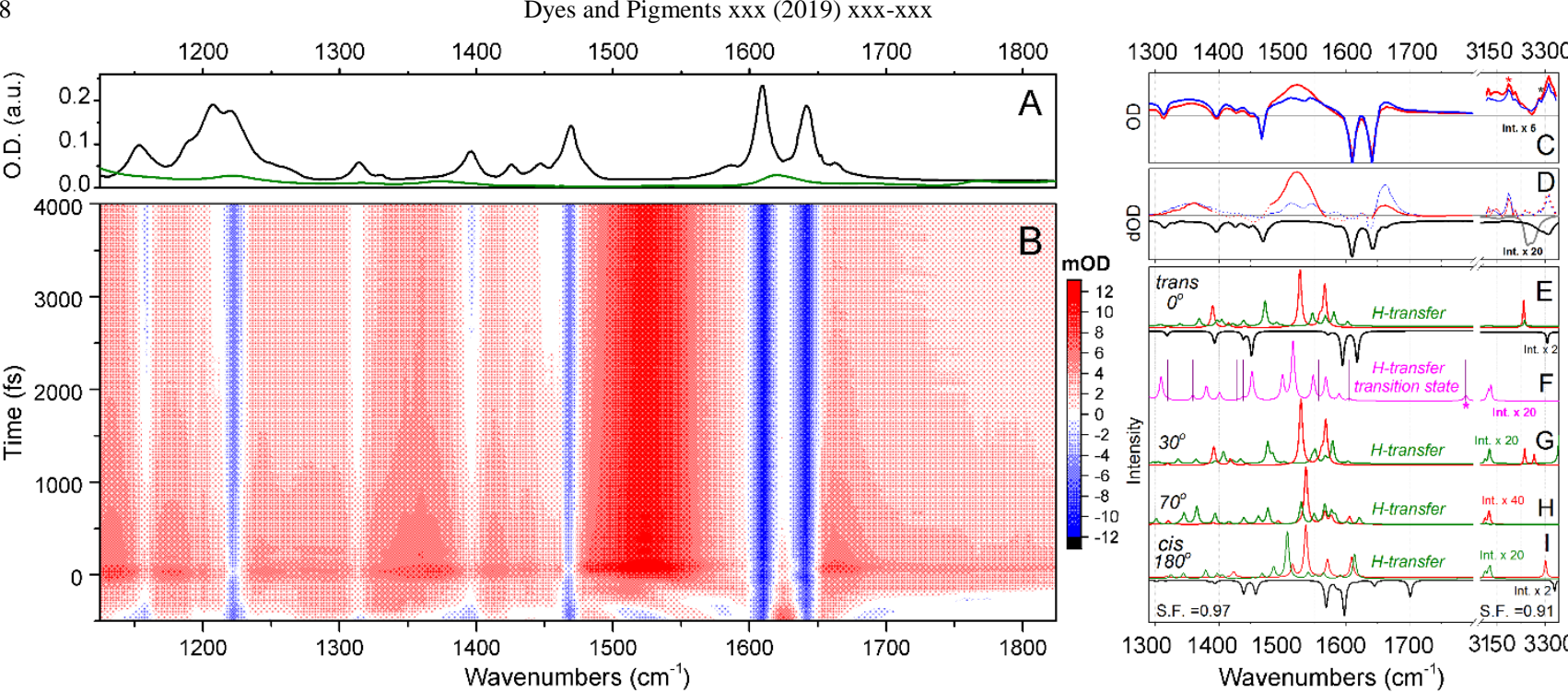

Fig. 5. Panel A: FTIR for solution of indigo carmine in deuterated DMSO (black line) deuterated DMSO alone (green line). Panel B: time resolved differential infrared response of indigo carmine in deuterated DMSO after excitation at $630 \mathrm{~nm}$. Panel C: differential infrared spectra at 0.1 (blue line) and 1 picoseconds (red line) delay times; Panel D: negative FTIR spectra of indigo carmine in deuterated DMSO (black line), blue and red lines show spectral features of the excited state absorption at 0.1 and 1 picoseconds which we factor out upon additions of scaled FTIR spectra to the time-resolved spectra as shown in Panel C (dotted regions indicate spectral regions complicated by heat); Panel E: calculated infrared absorptions of the ground state (black line), excited state (red line) and for the excited state where proton transfer is accounted for on one side (green line) of the molecule in the trans conformation. The spectral responses of the ground states are plotted as negative signatures to help discussion of the time-resolved responses. Panel F: calculated infrared absorptions of the transition state for proton transfer on one side of the trans chromophore when it is in the electronic excited state. Star labels the vibrational state which was considered in Ref. 23 to discuss the FT Raman signal at $1770 \mathrm{~cm}^{-1}$ reported there. Vertical lines indicate states which are anticipated to contribute into the Raman response if observed for such a structure. Panels G, H and I: the same results, according to the color code, as in Panel E, but for indigo carmine when under a $30^{\circ}$ twist from the trans geometry, when under a $70^{\circ}$ twist from the trans geometry and when in the cis conformation, respectively.

representative chromophore, when it is in the electronic excited state. In Fig. 5B we present the time-resolved infrared response of indigo carmine after excitation of the system by a pulse with its spectrum centred at $626 \mathrm{~nm}$. The presented time-resolved spectra are processed to remove chirp contributions: the original data affected by chirp is provided in the Supporting information file. We scan the response from -1 to $4 \mathrm{ps}$ with a time step of $20 \mathrm{fs}$. The detected time-dependent infrared response contains a number of spectral signatures, which have to be carefully addressed to distinguish the signs of the normal modes of the first electronic excited state, possible contributions of the solvent and spectral signatures of thermal dynamics, or redistribution of heat. The latter may include i) the spectral signatures of direct energy transfer to or between spectrally overlapping normal modes of the chromophore and solvent in case of anharmonic coupling, ii) spectral changes due to an offset of the Boltzmann distribution upon population of higher energy low frequency libration modes, iii) time-resolved effects of refractive index [37,38].

In Fig. 5C we show the IR responses at about 0.1 and 1 picosecond after the excitation in the visible: we may clearly see the relatively narrow bleaches of the infrared resonances at about $1311,1390,1470,1609,1640$ and $3290 \mathrm{~cm}^{-1}$ which we may assign to the reduction of the contributions of the normal modes of the chromophore in its electronic ground state upon its excitation by the pulsed radiation in the visible. Here, we may notice that there is a very broad positive off-set, which we assign to a long-term (quasi-constant) thermal background. This strongly affects the signals detected at 3 micron range. Exploring the recovery of the bleach at $1380 \mathrm{~cm}^{-1}$ (this spectral region is less complicated) we anticipate 88 picoseconds, which is consistent with results reported earlier anticipating the life-time of the first electronic excited state according to the results of a pump-probe experiment in the visible $[21,39]$.

Next, to show more clearly the infrared signatures of the chromophore in its first electronic excited state, in Fig. 5D, we present spectra where contributions of the bleaches are factored out (using FTIR of indigo carmine in its electronic ground state, Fig. 5A) and the broad long-term thermal off-set is subtracted. The subtractions are ambiguous in the spectral regions of fundamental IR transitions. To indicate these we used dotted lines. Exploring the spectra in Panels C and D we may identify several positive spectral features at about 1380, $1540 \mathrm{~cm}^{-1}, 1660$ and $3186 \mathrm{~cm}^{-1}$. The signature at $1660 \mathrm{~cm}^{-1}$ was reported previously [20]. In the spectral region $1510-1590 \mathrm{~cm}^{-1}$, the response at 0.1 picoseconds may be modeled with two states centered at 1514 and $1550 \mathrm{~cm}^{-1}$. Within several hundred femtoseconds, the lower and the higher energy resonances shift to 1517 and $1535 \mathrm{~cm}^{-1}$, respectively. If we exclude possible complications due to a coherent artifact (it should not contribute if spectral dynamics are present on a picosecond time-scale after the zero time), we may assign the very fast spectral dynamics in the spectral region $1510-1590 \mathrm{~cm}^{-1}$ to a structural optimization upon departure from the Franck-Condon region. Exploring the time decay of the spectral signature at 1540 $\mathrm{cm}^{-1}$ we anticipate the life-time of the normal mode of the chromophore to be 88 picoseconds while it is in its electronic excited state. This is consistent with the bleach recovery at 1380 $\mathrm{cm}^{-1}$ and the electronic excited state life time reported earlier [21,39].

At the high frequency side, as shown in Fig. 5C and 5D, we present the response in the 3 micron spectral range. In this spectral 
region, we expect contributions mainly due to antisymmetric $\mathrm{NH}$ stretching modes, as described Fig. 4B and C. TD-DFT studies of the $\mathrm{NH}$ normal modes of the cis conformer in the electronic excited state indicate that due to lower symmetry (the conformer is not planar due to electronic repulsion) both symmetric and antisymmetric NH stretching modes (Fig. 4D) contribute nearly equally in this spectral region, see Fig. 5I. The results of timeresolved experiments indicate that the response at 3 micron, in the bleach region, is complicated by the contribution of the solvent. However, using DFT predictions and the results of FTIR experiments, we may suggest that the subtle shoulder at $3287 \mathrm{~cm}^{-}$ ${ }^{1}$ (see the right star in Fig. 5C) is due to the $\mathrm{NH}$ antisymmetric stretching mode of the trans chromophore when in the electronic ground state. It is squeezed between the intense spectral signatures of the solvent. Due to the large lower frequency shift, as anticipated by TD-DFT, see Fig. 5E, we ascribe the obvious positive peak at $3186 \mathrm{~cm}^{-1}$ (see the left star in Fig. 5C) to the antisymmetric $\mathrm{NH}$ stretching mode of the trans chromophore, when in the electronic excited state (Fig. 4C).

To understand the nature of the normal modes that contribute to the IR response of indigo carmine in the electronic excited state, we used the results of TD-DFT theory for indigo carmine in trans and cis geometries: see the graphical presentations of the atomic displacements of the IR active vibrations in Fig. 4, and for possible structural variances with/without proton transfer dynamics in the electronic excited states. First, we focus on the properties of the chromophore in the trans geometry. For this, we may clearly see that the electronic excited state normal modes at 1567 and at 1528 $\mathrm{cm}^{-1}$ closely resemble those at 1617 and $1594 \mathrm{~cm}^{-1}$ for the electronic ground state, respectively. The red shift of the higher frequency vibration is relatively easy to interpret - it is consistent with the anticipated increase of the interatomic distances in the $\mathrm{C}=\mathrm{O}$ moieties when the chromophore is promoted to the first electronic excited state, see Table I.

The small shoulder at $1559 \mathrm{~cm}^{-1}$ in the calculated spectrum (red line in Fig. 5E) is due to the normal mode where simultaneous compressions of $\left(\mathrm{C}_{5}-\mathrm{C}_{6}\right)$ and $\left(\mathrm{C}_{3}-\mathrm{C}_{8}\right)$ bonds on one side of the chromophore is accompanied by simultaneous extension of $\left(\mathrm{C}_{5^{\prime}}\right.$ $\mathrm{C}_{6^{\prime}}$ ) and $\left(\mathrm{C}_{3^{\prime}}-\mathrm{C}_{8^{\prime}}\right)$ bonds on the other side of the chromophore (see the graphic in the set for the excited trans geometry in Fig. 4C). The corresponding vibration in the electronic ground state shows as a small peak at $1572 \mathrm{~cm}^{-1}$, see negative black line spectrum in Fig. 5E. Upon transition to the electronic excited state the lengths of the bonds $\mathrm{C}_{5}-\mathrm{C}_{6}$ and $\mathrm{C}_{5^{\prime}}-\mathrm{C}_{6^{\prime}}$ decrease from 1.411 to $1.408 \AA$ ($0.21 \%$ ), and the length of the bonds $\mathrm{C}_{3}-\mathrm{C}_{8}$ and $\mathrm{C}_{3^{\prime}}-\mathrm{C}_{8^{\prime}}$ increase from 1.415 to $1.423 \AA(+0.57 \%)$. It appears that the latter structural change dominates in the anticipated frequency shift for this mode when the chromophore is electronically excited. The frequency shift, however, is relatively small. Consequently, while this vibration is at the lower frequency side of the main doublet at 1617 and $1594 \mathrm{~cm}^{-1}$ when in the ground state, it shows weak infrared absorption on the red side of the higher frequency state of the main doublet at 1567 and $1528 \mathrm{~cm}^{-1}$ in the excited state.

Besides the modes we have discussed, in the spectral range from 1000 to $1800 \mathrm{~cm}^{-1}$, the calculations anticipate two more relatively strong vibrations for indigo carmine in the electronic excited state - at 1415 and at $1390 \mathrm{~cm}^{-1}$. The lower frequency vibration, at $1390 \mathrm{~cm}^{-1}$, describes mainly the in-plane $\mathrm{N}-\mathrm{H}$ and C$\mathrm{H}$ bendings, where on one side, if the $\mathrm{N}-\mathrm{H}$ and $\mathrm{C}_{7}-\mathrm{H}$ move towards the oxygen next to $\mathrm{C}_{2^{\prime}}$, then on the other side $\mathrm{N}-\mathrm{H}$ and $\mathrm{C}_{7}-\mathrm{H}$ move away from the oxygen atom next to $\mathrm{C}_{2}$. The higher frequency mode at $1415 \mathrm{~cm}^{-1}$ involves on one side of molecule an in-plane bendings of $\mathrm{C}_{6}-\mathrm{H}$ towards the proximal sulfonate groups with simultaneous stretching out of $\mathrm{C}_{4}-\mathrm{C}_{5}$ and $\mathrm{C}_{4}-\mathrm{C}_{3}$ bonds, at the other side the motions of the corresponding atoms are of opposite character. Their close analogues in the electronic ground state mode are the normal modes at 1451 and $1393 \mathrm{~cm}^{-1}$, respectively: see the indicated graphical presentation for the atomic displacements in Fig. 4B.

Interestingly, the calculated infrared properties of the first electronic excited state of trans indigo carmine cannot explain the observed optical absorption at $1660 \mathrm{~cm}^{-1}$. This is important to notice because this time-dependent absorption was reported before in tetramethyl indigo [21] and assigned as an infrared absorption of a $\mathrm{C}=\mathrm{O}$ stretching mode of the chromophore when in the first electronic excited state. In our experiments on indigo carmine, the decay of this spectral signature happens on a much faster time scale (within several picoseconds) while the electronic relaxation of the excited state is determined to be ca. 88 picoseconds, consistent with the results of a pump-probe experiment in the visible [21,39]. The theoretical studies conducted for indigo, 5,5'-dimethylindigo, indigo carmine, and thioindigo, 6-bromoindigo, 6,6'-dibromoindigo, 5-bromoindigo (data not shown), 5,5'-dibromoindigo (data not shown) and for indigo carmine indicate that for all these molecules (while in the trans geometry) the excited state absorption of the normal modes in the considered spectral regions experience red frequency shifts compared to the frequencies of the corresponding normal modes when the chromophores are in the electronic ground state. The experimental and theoretical results may suggest that the optical absorption at $1660 \mathrm{~cm}^{-1}$ is a non-equilibrium heat contribution due to the solvent as it has a very weak optical absorption in this spectral region. Alternatively, we may consider the possible contribution of a structural state associated with either torsional or proton transfer dynamics in the electronic excited state. To explore this, in Fig. 5E-5I using red lines we plot infrared spectra for indigo carmine in the electronic excited state when the central C-C bond allows a possible rotation of the planes of the two of the normal modes in the ground and in the excited states of the $30^{\circ}$ and $70^{\circ}$ twist (from trans) conformers and cis conformer of indigo carmine. Spectral properties of the excited states under twisted geometry do not explain well the short living spectral specie at $1660 \mathrm{~cm}^{-1}$.

Next, using green lines we show infrared spectra for the same structures but when the proton is allowed to be transferred on one side of the molecule. The spectral signatures of the $30^{\circ}$ and $70^{\circ}$ conformers (red lines in Panel $\mathrm{G}$ and $\mathrm{H}$ ) do not differ significantly from that of the model molecule in the trans geometry. It is interesting to notice that the infrared absorption of the $70^{\circ}$ conformer in the excited state, where proton transfer is accounted, see green line in panel $\mathrm{H}$, may explain the experimentally observed spectral signature at $1660 \mathrm{~cm}^{-1}$.

Since time-resolved Fourier transform Raman spectroscopy reported in [23] suggested the possible, spectral behavior of a transition state for a proton transfer on one side of the molecule 
when in the electronic excited state here, additionally, in panel $\mathrm{F}$, we present the calculated response for such a structure. With the vertical lines in the panel we indicate the normal modes expected to contribute into the Raman response. One of the calculated resonances (see star label in the panel), indeed, is proximal to the observed response at $1750 \mathrm{~cm}^{-1}$ [23]. The other activities were not reported using FT time-windowing of modulations of the visible ultrafast responses induced by $5 \mathrm{fs}$ pulses under fluences of 2580 and $480 \mathrm{GW} / \mathrm{cm}^{2}$ [23]. Reviewing the results for the excited state of the cis conformer, Fig. 5I, we see that the infrared spectrum of the electronic excited state also does show a spectral signature at $1610 \mathrm{~cm}^{-1}$, which could be considered as a candidate for the relatively short-lived absorption observed in experiment at 1660 $\mathrm{cm}^{-1}$. At the same time, the experimental data indicates that the role of the cis conformer in the ground state is negligible - we do not see in FTIR nor in time-dependent responses any features, which could be specific to the relatively red-shifted (compared to such for the trans conformer) strong resonances of the ground state fundamentals of the cis conformer at 1570 and $1600 \mathrm{~cm}^{-1}$ as shown in Fig. 5G. Also, we do not see a high frequency bleach contribution above $1650 \mathrm{~cm}^{-1}$, which would be specific to the symmetric carbonyl stretch of the cis conformer and should be present if such geometry would play a significant role. Nevertheless, it is still possible that upon excitation in the visible, the change of the electronic nature of the chromophore and the nature of the central $\mathrm{C}-\mathrm{C}$ bond would allow a very fast flip from trans to cis configuration for some of the molecules.

It is interesting that the frequency separation and the intensity difference between the anticipated resonances at 1516.1 and $1557.3 \mathrm{~cm}^{-1}$ for the earlier time delay (note, the lower frequency peak is nearly ten times more intense than the higher frequency one) also corresponds better to the calculated infrared response of the cis conformer in its first electronic excited state. If such is the case, the time-scale (less than 1 picosecond) of the spectral dynamics in the spectral region $1510-1590 \mathrm{~cm}^{-1}$ would heterocyclic indole ring systems in respect to each other.

To understand this better we conduct DFT and TD-DFT calculations suggest an immediate, ultrafast recovery of the trans configuration of the molecular geometry after photo-excitation to the first electronic excited state. Also, this would help to explain the very fast decay of the optical density at $1660 \mathrm{~cm}^{-1}$ : see blue and red line spectra in Fig. 5D. This would correspond to the very fast loss of the spectral signature of the state which would resemble the symmetric carbonyl vibration which theory anticipates for the electronic excited state of cis indigo carmine at $1609 \mathrm{~cm}^{-1}$ : see Fig. 4D and 5I. The anticipated role of the cis geometry in the electronic excited state cannot be dominant, as it would not explain the optical density at $1360 \mathrm{~cm}^{-1}$, which experiment reports at the very early time. We may ascribe this optical density to the contribution of the vibration which theory anticipates for the electronic excited state of trans indigo carmine at $1390 \mathrm{~cm}^{-1}$ : see Fig. 4C and 5E.

In result, we may state that at $0.8-1$ ps the experiment demonstrates optical absorption properties which can be ascribed to the trans geometry molecule in the first electronic excited, save the small residual positive optical absorptions at $1660 \mathrm{~cm}^{-1}$. This spectral signature, however, decays much faster than the

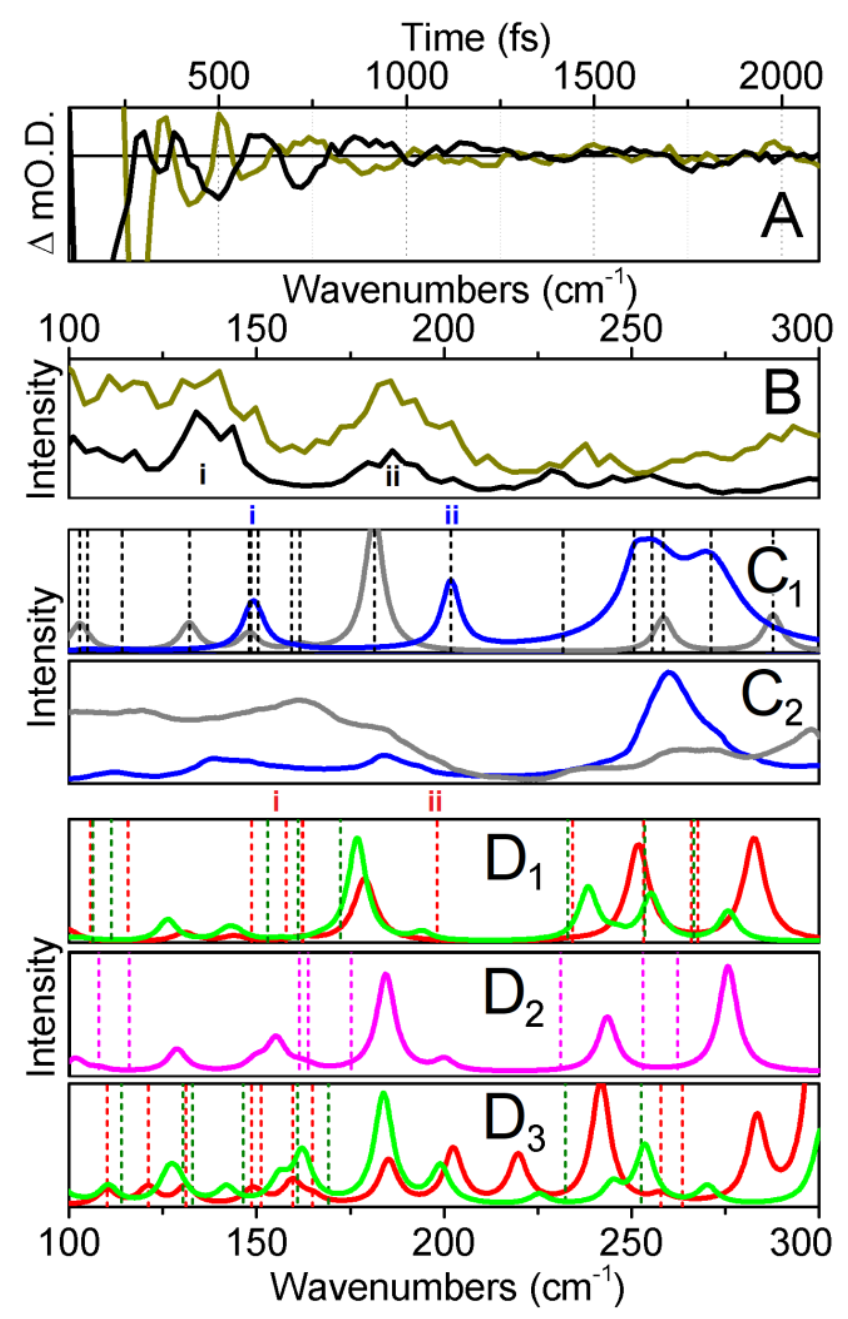

Fig. 6. Panel A: kinetics of the residual oscillations after subtraction of the population decay and heat decay contribution from the time dependent response in the spectral ranges of the excited state responses at 1550 (black line) and $3200 \mathrm{~cm}^{-1}$ (orange line). Panel B: Fourier transform of the residual oscillations detected at 1550 (black line) and $3200 \mathrm{~cm}^{-1}$ (orange line). Panel C1: infrared (grey line), and pre-resonant Raman (blue line) spectra calculated for indigo carmine in the ground electronic state; black dashed lines indicate calculated resonances. Panel C2: FTIR spectrum of solid indigo carmine (grey line), and resonant Raman spectrum (magenta line) of indigo carmine solution in deuterated DMSO detected using excitation at $532 \mathrm{~nm}$. Panel D1: infrared spectra calculated of trans indigo carmine in the electronic excited state (red line) and when proton transfer happens on one side of the molecule. Panel D2: infrared spectra calculated for the transition state (saddle point) for proton transfer on one side of trans indigo carmine in the electronic excited state. Panel D3: results of analogous calculations as in D1 but for cis indigo carmine in the electronic excited state. Dashed lines in panels D indicate calculated resonances which have low IR absorption and, hence, may contribute to strong Raman.

life-time of the electronic excited decay. Considering theory predictions, we may suggest three scenarios: i) the signature may be due to a solvent non-equilibrium thermal response, ii) this could be due to spectral contribution of some chromophores that experience proton transfer in the excited state, iii) this may be due to the symmetric carbonyl stretching of a cis-like short lived structural state. To sort the suggested scenarios, next, we explore low Fourier transformed modulations of the IR optical absorption in the electronic excited state and we review the energetics of the considered processes. 
3.4. Structure and dynamics in the electronic excited state: low frequency coherences

In our Visible Pump - infrared Probe experiment we employ coherent excitation radiation with the spectral envelope centred at $626 \mathrm{~nm}$ with full width at half maximum of $14 \mathrm{~nm}$, which corresponds to a spectral acceptance window of $360 \mathrm{~cm}^{-1}$. The width allows sampling to see if low frequencies (within the provided bandwidth) would modulate the potential surface of the prepared infrared coherences. Specifically, we explore Fourier transform of time-resolved oscillations of infrared absorption of the normal modes of the molecule in the first electronic excited state, see Fig. 6A. The recurrences are extracted subtracting the time-dependent component specific to relaxation of the electronic excited state from the detected response [40]. It is striking to see that the residual time sampled oscillations at 1550 and $3200 \mathrm{~cm}^{-1}$, which we ascribe to $\mathrm{C}=\mathrm{O}$ and $\mathrm{NH}$ stretching modes of the chromophore when in the first electronic excited states, are $\pi / 2$ shifted.

In Fig $6 \mathrm{~B}$ we present the Fourier transform spectra of the recurrences. The higher the frequency of the observed spectral component the lower its intensity, according to the finite bandwidth of radiation used for preparation of the involved coherences. We may sort the calculated low frequency normal modes (of trans indigo in the ground state) into three groups according to their spectral regions: i) the states at 132, 148 and $149 \mathrm{~cm}^{-1}$ would belong to the first group; ii) the state at $182 \mathrm{~cm}^{-1}$ to be considered alone; iii) the vibrations at $232 \mathrm{~cm}^{-1}, 256$ and 258 $\mathrm{cm}^{-1}$ would belong to the third group. Low frequencies at 256 and $258 \mathrm{~cm}^{-1}$ that modulate time-resolved nonlinear responses of indigo carmine were reported previously and were obtained using ultrafast Visible pump - Visible probe spectroscopy [39]. The spectral signatures were assigned to in-plane and out-of-plane bending modes. Here, it is important to note that time resolved modulations of electronic responses are sensitive to Raman activities via electronic transitions between the ground and various electronic excited states according to the bandwidth of the refractive part of the nonlinear response function. In our studies, taking advantage of spectral selectivity in the infrared, we extract low frequency coherences which are specific to vibrations of the structure in the first electronic excited state. According to our knowledge, this is the first observation of low frequency coherences modulating selected infrared responses of indigo in the first electronic excited state, see Fig 6B.

Calculation of high quality second-order derivatives for molecules in the electronic excited state is beyond the current precision of TD-DFT theory [41]. Accordingly, to approach the nature of low frequency Raman modulations involved in relaxation when in the electronic state, first, we compare the FT spectra of time resolved modulations with calculated and experimentally measured infrared and Raman responses of indigo carmine in the ground electronic state, see Fig. $6 \mathrm{C}_{1}$ and $6 \mathrm{C}_{2}$, respectively. The theoretical and experimental pre-resonant Raman responses for the system in the ground state correspond well to the spectral signatures from the system in the electronic excited state, though they are slightly spectrally shifted. Complicated by intermolecular interactions, nonetheless, FTIR in the low frequency region does overall agree with the

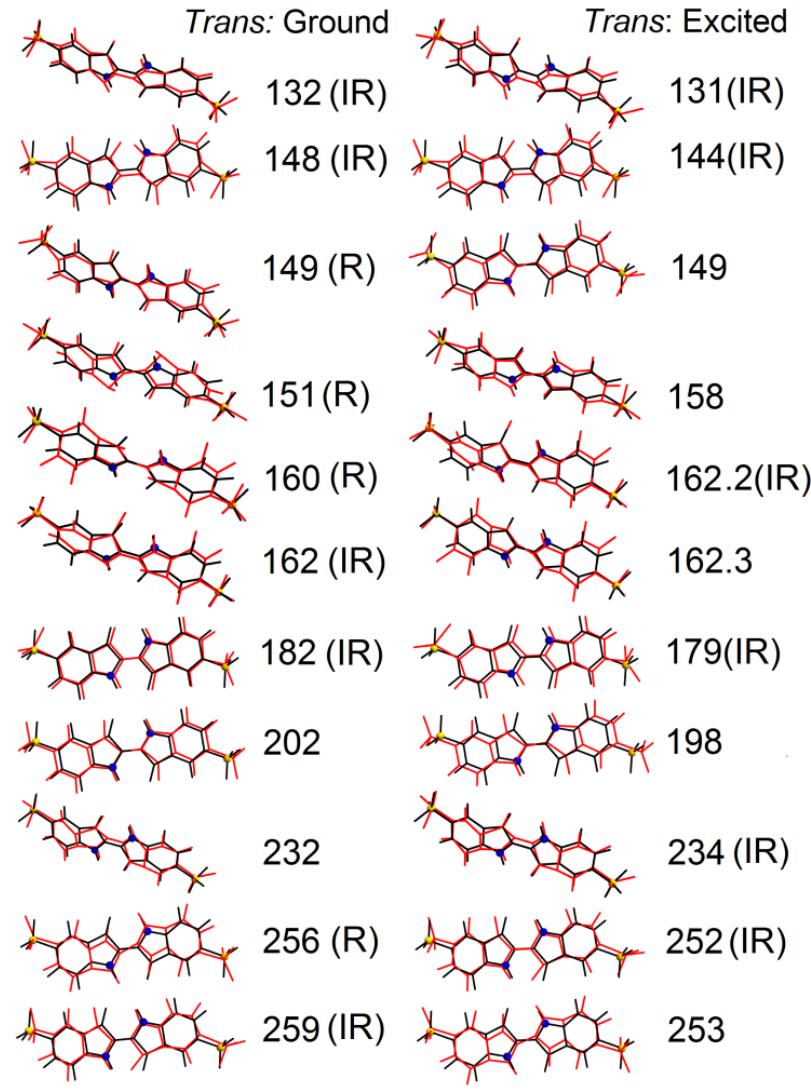

Fig. 7. Graphical representations of the infrared and Raman active norma modes (IR and $\mathrm{R}$ indicated, respectively) of trans indigo carmine in the electronic ground and excited state, as indicated. The scaling factor for the calculated frequencies is 1 .

theory prediction and with the spectral densities below $200 \mathrm{~cm}^{-1}$ and above $250 \mathrm{~cm}^{-1}$ as shown if Fig. 6B. At this point, it is helpful to present and examine the atomic displacements of the low frequency normal modes of trans indigo carmine when in the ground electronic state: see Fig. 7. Theory suggests that experimentally observed pre-resonant Raman activities in the ground state at 149 and $151 \mathrm{~cm}^{-1}$ include both in-plane and out-ofplane deformations, though the former dominate. In both cases, Hbonds experience strong symmetric stretchings on both sides of the molecule. Resonant Raman mode at $202 \mathrm{~nm}$ involves in-plane activities only. For this mode, symmetric stretchings of $\mathrm{H}$-bonds play a major role. As we may see, in the left side of Fig. 7, the corresponding modes for indigo carmine in the electronic excited state are at 149,158 and $198 \mathrm{~cm}^{-1}$ : see graphical presentations in Fig. 7 and the corresponding red dashed line indicators in Fig. $6 \mathrm{D}_{1}$. Due to the asymmetry, we may not expect a significant Raman contribution from the mode at $200 \mathrm{~cm}^{-1}$ for indigo carmine when a proton is transferred on one side of the molecule: note the lack of the dashed green line in Fig. 6D1. Consistently, the normal modes of excited state indigo carmine under $30^{\circ}, 70^{\circ}$ and $180^{\circ}$ (cis form) torsion demonstrate relatively strong IR absorptions in the spectral range between 170 and $250 \mathrm{~cm}^{-1}$ : for example, see green line spectrum in Fig. $6 \mathrm{D}_{3}$. The same lack of symmetry makes the normal modes in the considered spectral region for excited state indigo carmine where proton transfer on one side would experience the saddle point in the structural region of the transition state rather Raman inactive: Fig. 6D2. 


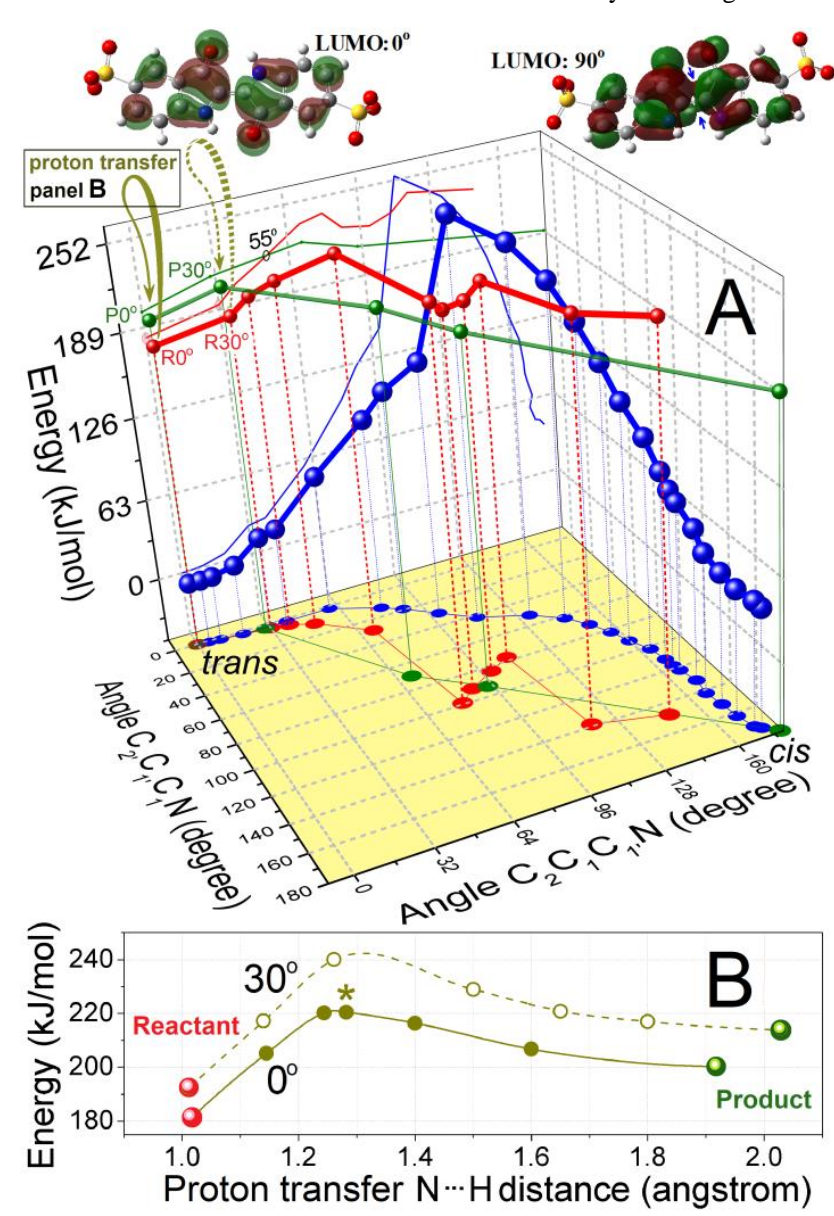

Fig. 8. Panel A: potential energy of indigo carmine in its ground (blue circle line), in the first electronic excited state (red circle line) and for the molecule in the electronic excited state when proton transfer is accounted on one side (green line) in dependence on CCCN angles. Here, we constrained only the $\mathrm{C}_{2} \mathrm{C}_{1} \mathrm{C}_{1} \mathrm{~N}$ angle to the values as indicated and optimized structures to extract energies in the electronic ground (blue circled line) and excited (red circled line) states. Upon the conducted optimization the second angle $\mathrm{C}_{2} \mathrm{C}_{1} \mathrm{C}_{1} \mathrm{~N}$ was allowed to adjust. Panel $\mathrm{B}$ : energy pathways for proton transfer on one of indigo carmine sides when the chromophore is in the electronic excited state under trans and $30^{\circ}$ twisted geometry, as specified, Star indicates theoretically anticipated saddle point for the trans geometry that was used to calculate normal modes as described in Fig. 5F.

Beside the considered proton transfer dynamics, FT low frequency spectroscopy should be able to report on photo-induced isomerization of indigo carmine as well. Spectral properties calculated for the cis indigo carmine, when in the electronic excited state shown using the red line in Fig. $6 \mathrm{D}_{3}$ do not agree with the experimental results, as shown in Fig. 6B. The disagreement provides a strong suggestion that photo-induced isomerization does not happen for indigo carmine in the first excited state in DMSO environment. At the same time, we may suggest reasons for the lack of obvious experimental evidence to report on possible products either along the proton transfer reactive pathway [22,23] or along a photo-induced isomerization pathway. First, it is possible to think that while using hundred femtosecond pulses the time of decay for the mentioned species is faster than the bandwidth for the necessary recurrence time. Second, the lack of coherence, also, may be explained if dynamics explore a chaotic regime as was reported recently [42], for a $\mathrm{C}-\mathrm{N}-\mathrm{K}$ molecule allowed to pass over the saddle point in a two-dimensional potential surface with two local minima. It is also obvious, that time-resolved infrared absorption experiments do not have the sensitivity necessary to report on analytical presences, which can be confirmed using fluorescence photo-counting in the visible [22].

\subsection{Mechanisms of effective relaxation}

What are the mechanisms for indigo photo-stability? In earlier studies [16], it was suggested that Hydrogen bonding between the two adjacent carbonyl and $\mathrm{NH}$ groups would keep the molecule in its trans planar configuration. Later, proton dynamics and proton transfer were discussed to describe results of optical spectroscopy in the visible $[22,23]$. The results of our time resolved IR studies do suggest that in the Franck-Condon region indigo may explore torsional dynamics outside of the structural region, which is optimal for Hydrogen-bonding. At the same time, our results in the mid-IR may account for the possible contributions of torsional states where proton displacement, as was discussed before in [23], may happen on one side of the molecule. To understand these better, here, we review such processes from the perspective of energetics. In Fig. 8 we present DFT and TD-DFT anticipations of potential surfaces scanned along the isomerization degree of freedom between the cis and trans states in the electronic ground and excited states (see red and blue lines in panel A), and in the case if a proton transfer takes place in the electronic excited state (see green and dark yellow lines in panels A and B respectively).

First, let us consider the torsional degree of freedom: the results (see blue and red lines in Fig. 8A ) indicate that there is a stabilizing effect for the trans geometry both, in the ground state and in the electronic excited states due to Hydrogen bonding: in both states the energies of the trans form are about $50 \mathrm{~kJ} / \mathrm{mol}$ lower than the energy of the cis states. We see that regardless of the expected electric repulsion between the $\mathrm{C}=\mathrm{O}$ and $\mathrm{NH}$ moieties, the potential energy surface has a rather planar character, when the system is suggested to depart from the cis geometry in the excited state. At the same time, we must explain the very high barrier in the ground state and the obviously much smaller but still present barrier in the excited state. The main reason for such are the unfavourable interactions between the components of the wavefunctions located on the two rings if explored along the isomerization degree of freedom. Specifically, when the system is in the ground state, a departure from the trans geometry towards the transition state leads to a reduction of electronic delocalization of the HOMO (see Fig. 2B). When the system is in the excited state, a departure from the trans geometry would lead to interactions between the components of the LUMO, which are of the same sign but located on different indole rings: see the contact regions indicated by blue arrows for $90^{\circ}$ LUMO, Fig. 8. Such interactions would be unfavourable as the wave-function is of an anti-bonding character. When the system is in the valley of the trans form it finds additional stabilization due to optimal Hydrogen bonding. When it is in the valley of the cis form the energy of the conformer is higher and the system departs from being very planar as there is a slight electronic repulsion.

The description above does not yet explain if and why indigo chromophores would "prefer" to be statistically on the side of the trans form. According to Fig. 8A, there are two predictions by TD- 
DFT, which may help explain this. First, we may notice that upon departure from the cis geometry in the electronic excited state the energy pathway has overall a reactive character on moving towards the trans geometry. In other words, the barrier from that side is rather small. Second, since the predicted barrier in the electronic ground state is rather high, in the region of the transition state, also, there is a non-negligible probability of crossing between the potential surfaces of the electronic excited and ground states. In other words, our theoretical studies provide that, with a small surplus due to kinetic energy, the system can follow a conical intersection pathway of relaxation. Such a mechanism is being explored to explain several photodynamic observations and effective electronic relaxation pathways in organic and biological systems [43-46].

Second, let us describe possible effects due to proton transfer. For the reactant, transition state and photo-product indigo carmine under trans geometry in the electronic energies (in the FranckCondon region) theory anticipates energies of 181.4, 220.5 and $200.2 \mathrm{~kJ} / \mathrm{mol}$, respectively. The cost to pass over the barrier there is about twice higher than that reported previously [23]. The difference is likely due to the fact that we accounted solvent in our calculations using PCM. Here, it is important to note (see Fig 8B) that for the considered proton transfer dynamics passing over the barrier is even less likely if indigo carmine departs from the trans geometry. The results suggest that the probability of proton transfer in the Franck-Condon region should be small if not negligible. Of course, we cannot rule out the possibility that under high power excitation, either due to coherent phenomena or due to non-equilibrium heating, proton transfer may be stimulated. However, it important to note that our theory studies, Fig. 8A, predict that once the torsion of the structure in the excited state is above $55^{\circ}$, energy of the product for proton transfer becomes lower than that for the reactant. This does provide a reason to expect that fluorescence photon-counting may report on proton tunnelling [22]. If we assume that in our experiment, under the power of the visible radiation we used, some of trans indigo carmine molecules would undergo proton transfer in the FranckCondon region with consequent torsional dynamics, then the relatively short living spectral signature at $1660 \mathrm{~cm}^{-1}$ may be assigned to a minor contribution of the admixing of $\mathrm{C}-\mathrm{OH}$ and $\mathrm{C}$ $\mathrm{NH}$ bends with the stretching of the central $\mathrm{C}-\mathrm{C}$ bond. The lifetime of such structural states would be short and the description would resemble that in [23] save we accounted torsion after proton displacement.

Our experimental results, with the support of DFT calculations, indicate that upon photo-excitation the main pathway of energy relaxation of an indigo chromophore would conserve its trans geometry without any proton transfer. Furthermore, we may suggest that the planarity of the structure would be necessary to facilitate effective energy dumping via coupling between the inplane delocalized stretching and bending vibrational admixtures which cascade from higher to lower frequency manifolds of vibrational down to libration states and bath modes. In this picture, not letting the chromophore "wander" from the trans geometry may play a central role in effective relaxation.

\section{Conclusions}

Indigo and its derivatives are among the most ancient dyes of bio-organic origin. Some of the related pigments demonstrate remarkable photo-stability for hundreds of years $[12,47]$. Variance in chemistry of the side groups at different ring positions promotes significant diversity in the energies of the electronic transitions in the UV-VIS spectral region. In this study, first, using UV-VIS, non-resonant Raman, resonant Raman and FTIR spectroscopy, we explore the photo-physical and structural properties in a series of indigo pigments and chromophores of natural and synthetic origin, respectively. Most of the spectral characterizations for the samples of natural origin are reported here for the first time. Second, assisted with the predictions of DFT for a series of model indigo molecules, we compare the detected electronic, Raman and infrared responses of the pigments and chromophores. According to the results, we select indigo carmine solution in DMSO as a model system to study the photochemistry of such chromophores and the mechanisms of their photo-stability. Specifically, we explore the structure and dynamics of indigo carmine in its first electronic excited state using femtosecond Visible Pump - infrared Probe spectroscopy. Ultrafast experiments indicate that even if a departure from trans geometry happens upon photoexcitation in the visible, the normal modes of the trans geometry of the electronic excited state are reconfirmed within several hundred femtosecond after photoexcitation.

The novelty here is in using time-resolved mi-IR spectroscopy to probe directly the spectral signatures of the molecular vibrations when in the first electronic excited state and specifically to compare the data with predictions by TDDFT. The suggested analysis is extended to the low frequency manifold using FT of the time-resolved modulations of IR absorption in the electronic excited state. Another new aspect is that we reviewed the results of the experimental studies accounting for the energetics of possible reactivity in the electronic excited state along both, torsional and proton transfer reactive pathways. The results of experimental steady-state and time-resolved infrared studies conducted here indicate a cascade of in-plane delocalized normal modes in mid-IR spectral range to assist energy redistribution towards low frequency modes. Sampling the low frequency vibrations of the coherent modulates of infrared responses specific to the first electronic excited state, we show that contributions of the delocalized in-plane skeletal C-C stretching and C-C-C bending modes dominate in relaxation. One of the contributing coherences, at $198 \mathrm{~cm}^{-}$ 1 , indicates admixing of the intramolecular Hydrogen bond dynamics into the indicated in-plane vibrations.

Using TD-DFT theory we explored potential energies along the isomerization pathways of indigo carmine in the electronic excited and ground states. The results of quantum chemistry indicate that both the reactive character of the isomerization pathway (starting from the cis geometry) in the excited state and a non-negligible probability of conical intersection crossing due to a high barrier along the isomerization pathway in the ground state would provide for the dominance of the trans geometry of the chromophore in 
a photodynamic regime. The anticipated energetics are mainly due to the components of the electronic wavefunctions in the electronic ground and excited states, while intermolecular Hydrogen bonding plays a secondary, stabilizing role. In this picture of effective relaxation, suggested by both experiment and theory for indigo chromophores, we anticipate the planarity of the dominant trans geometry would provide effective dissipation of surplus energy in the vibrational manifold via the cascade of effectively coupled in-plane delocalized bending and stretching modes.

\section{Acknowledgments}

Dr. Victor V. Volkov and D. Carole C. Perry gratefully acknowledge funding from AFOSR FA9550-16-1-0213; thank Dr. Baruch Sterman - co-founder of the Ptil Tekhelet, Jerusalem, for providing crushed glands from Murex trunculus; and thank Dr. Joanna Aizenberg, Harvard University, for continued access to the Odyssey cluster at Harvard University. The computations in this paper were run on the Odyssey cluster supported by the Faculty of Arts and Sciences division, Research Computing Group at Harvard University. Dr. Victor V. Volkov and D. Carole C. Perry are thankful to Dr. Lufeng Zou, Dr. Fernando R. Clemente and Dr. Douglas J. Fox (Gaussian Inc., Wallingford, CT 06492 USA) for helpful discussions on structural optimizations in electronic excited states.

\section{References}

[1] Sadler P. Ultra-violet and infra-red spectra of substituted isoindigos and indirubins. Spectrochim Acta 1960;16:1094-1099. https://doi.org/10.1016/0371-1951(60)80150-6.

[2] Sadler P. Absorption Spectra of Indigoid Dyes. J Org Chem 1956;21:316-318. DOI: $10.1021 /$ jo01109a014

[3] Klessinger M, Lüttke W. Theoretische und spektroskopische untersuchungen an indigo-farbstoffen - II: Das chromophore system der Indigo-Farbstoffe Tetrahedron 1963;19:315-335. https://doi.org/10.1016/S0040-4020(63)80023

[4] Giustetto R, Xamena F, Ricchiardi G, Bordiga S, Damin A, Gobetto R, Chierotti M. Maya Blue: A Computational and Spectroscopic Study. J Phys Chem B 2005;109:19360-19368. DOI: 10.1021/jp048587h.

[5] Perpete E, Preat J, Andre J-M, Jacquemin D. An ab Initio Study of the Absorption Spectra of Indirubin, Isoindigo, and Related Derivatives. J Phys Chem A 2006;110:5629-5635. DOI: 10.1021/jp060069e.

[6] El-Mansy M. Quantum chemical studies on structural, vibrational, nonlinear optical properties and chemical reactivity of indigo carmine dye. Molecular and Biomolecular Spectroscopy 2017;183:284-290. https://doi.org/10.1016/j.saa.2017.04.047.

[7] Cooksey C, Tyrian Purple: 6,6'-Dibromoindigo and Related Compounds. Molecules 2001;6:736-769. DOI:10.3390/60900736.

[8] McGovern P, Michel R. Royal Purple Dye: Tracing Chemical Origins of the Industry. Anal Chem 1985;57:1514A-1522A. DOI: 10.1021/ac00291a753.

[9] Jackson J. The Geographical Distribution of the Shell-Purple Industry. Longmans: Green and Co: London; 1917;1-29.

[10] Cardon D. Natural Dyes. Archetype Publications Ltd.; 2007.

[11] Edens C. Khor Ile-Sud, Qatar: The Archaeology of Late Bronze Age Purpledye Production in the Arabian Gulf. Iraq, 1999;61:71-88.

[12] James M, Reifarth N, Mukherjee A, Crump M, Gates P, Sandor P, Robertson F, Pfälzner P, Evershed R. High prestige Royal Purple dyed textiles from the Bronze Age royal tomb at Qatna, Syria. Antiquity 2009;83:1109-1118. https://doi.org/10.1017/S0003598X00099397.
[13] Pliny. Natural History, Translated by H. Rackham; Loeb Classical Library 394 Cambridge, MA: Harvard University Press, 1952; Volume IX: Book 35: paragraph 46.

[14] Asiaticus. The Rise and Fall of the Indigo Industry in India. The Economic Journal 1912;22:86:237-247.

[15] Baeyer A, Drewsen V. Darstellung von Indigblau aus Orthonitrobenzaldehyd Berichte der Deutschen Chemischen Gesellschaft 1882;15:2856-2864. https://doi.org/10.1002/cber.188201502274.

[16] Brode W, Pearson E, Wyman G. The Relation between the Absorption Spectra and the Chemical Constitution of Dyes. XXVII. cis-trans Isomerism and Hydrogen Bonding in Indigo Dyes. J Am Chem Soc 1954;76: 1034 1036. DOI: $10.1021 /$ ja01633a033.

[17] Tilocca A, Fois E. The Color and Stability of Maya Blue: TDDFT Calculations. J Phys Chem C 2009;113:8683-8687. DOI 10.1021/jp810945a.

[18] Bernardino N, Brown-Xu S, Gustafson T, de Faria D. Time-Resolved Spectroscopy of Indigo and of a Maya Blue Simulant. J Phys Chem C 2016;120:21905-21914. DOI: 10.1021/acs.jpcc.6b04681.

[19] Kobayashi T, Rentzepis P. On the picosecond kinetics and photostability of indigo and 6,6'-dimethoxyindigo. J Chem Phys 1979;70:886. https://doi.org/10.1063/1.437479

[20] Elsaesser T, Kaiser W, Luttke W. Picosecond spectroscopy of intramolecular hydrogen bonds in 4,4',7,7'-tetramethylindigo. J Phys Chem 1986;90:2901-2905. DOI: 10.1021/j100404a024

[21] de Melo S J, Moura A P, Melo M. Photophysical and Spectroscopic Studies of Indigo Derivatives in Their Keto and Leuco Forms. J Phys Chem A 2004;108:6975-6981. DOI: 10.1021/jp049076y.

[22] Pina J, Sarmento D, Accoto M, Gentili P L, Vaccaro L, Galvao A, de Melo J S S. Excited-State Proton Transfer in Indigo. J Phys Chem B 2017;121:2308-2318. https://doi.org/10.1021/acs.jpcb.6b11020.

[23] Iwakura I, Yabushita A, Kobayashi T. Transition State in a prevented proton transfer observed in real time. Bull Chem Soc Jpn 2011;84:164-171. https://doi.org/10.1246/bcsj.20100269.

[24] Kaindl R, Wurm M, Reimann K, Hamm P, Weiner A, Woerner M. Generation, shaping, and characterization of intense femtosecond pulses tunable from 3 to $20 \mu \mathrm{m}$. J Opt Soc Am B 2000;17:2086-2094. https://doi.org/10.1364/JOSAB.17.002086.

[25] Bredenbeck J, Hamm P. Versatile small volume closed-cycle flow cell system for transient spectroscopy at high repetition rates. Rev Sci Instrum 2003;74:3188. https://doi.org/10.1063/1.1574605.

[26] Becke A. Density-functional exchange-energy approximation with correct asymptotic behavior. Physical Review A 1988;38:3098-3100. https://doi.org/10.1103/PhysRevA.38.3098.

[27] Lee C, Yang W, Parr R. Development of the Colle-Salvetti correlationenergy formula into a functional of the electron density. Physical Review C 1988;37:785-789. https://doi.org/10.1103/PhysRevB.37.785.

[28] Frisch M, Trucks G, Schlegel H, Scuseria G, Robb M, Cheeseman J, Scalmani G, Barone V, Mennucci B, et al. Gaussian 09, revision B.01; 2010

[29] Amovilli C, Barone V, Cammi R, Cancès E, Cossi M, Mennucci B, Pomell C, Tomasi J. Recent Advances in the Description of Solvent Effects with the Polarizable Continuum Model. Adv Quantum Chem 1998;32:227-261. https://doi.org/10.1016/S0065-3276(08)60416-5

[30] Jacquemin D, Le Bahers T, Adamo C, Ciofini I. What is the "best" atomic charge model to describe through-space charge-transfer excitations? Phys Chem Chem Phys 2012;14:5383-5388. DOI: 10.1039/C2CP40261K.

[31] Senju T, Mizuguchi J. Electronic Structure of Thiazine-Indigo Pigment on the Basis of the Crystal Structure. J Phys Chem B 2005;109:7649-7653. DOI: $10.1021 / \mathrm{jp} 0500510$

[32] Jacquemin D, Preat J, Wathelet V, Fontaine M, Perpete E. Thioindigo Dyes: Highly Accurate Visible Spectra with TD-DFT. J Am Chem Soc 2006;128:2072-2083. DOI: 10.1021/ja056676h.

[33] Wouters J, Verhecken A. Composition of Murex dyes. Coloration Technology 1992;108:404. https://doi.org/10.1111/j.14784408.1992.tb01489.x.

[34] Peica N, Kiefer W. Characterization of indigo carmine with surface enhanced resonance Raman spectroscopy (SERRS) using silver colloids and island films, and theoretical calculations. J Raman Spectrosc 2008;39:47-60. https://doi.org/10.1002/jrs.1813. 
[35] Ajiki H, Pozzi F, Huang L, Massa L, Leona M, Lombardi J. Raman spectrum of monobromoindigo. J Raman Spec 2012;43:520-525. DOI: 10.1002/jrs.3066.

[36] Amat A, Rosi F, Miliani C, Sgamellotti A, Fantacci S. Theoretical and experimental investigation on the spectroscopic properties of indigo dye. Journal of Molecular Structure 2011;993:43-51. https://doi.org/10.1016/j.molstruc.2010.11.046.

[37] Bredenbeck J, Helbing J, Kumita J, Woolley G, Hamm P. $\alpha$-Helix formation in a photoswitchable peptide tracked from picoseconds to microseconds by time-resolved IR spectroscopy. Proc Nat Acad Sci 2005; 102:2379-2384. https://doi.org/10.1073/pnas.0406948102.

[38] Park S, Nguyen P, Stock G. Molecular dynamics simulation of cooling: Heat transfer from a photoexcited peptide to the solvent J Chem Phys 2009; 131:184503. https://doi.org/10.1063/1.3259971.

[39] Nagasawa Y, Taguri R, Matsuda H, Murakami M, Ohama M, Okada T, Miyasaka $\mathrm{H}$. The effect of hydrogen-bonding on the ultrafast electronic deactivation dynamics of indigo carmine. Phys Chem Chem Phys 2004;6:5370-5378. DOI: 10.1039/B409443C.

[40] Rini M, Kummrow A, Dreyer J, Nibbering E, Elsaesser T. Femtosecond mid-infrared spectroscopy of condensed phase hydrogen-bonded systems as a probe of structural dynamics. Faraday Discussion 2002;122:27-40. DOI: $10.1039 / \mathrm{B} 201056 \mathrm{~A}$.

[41] communications with Gaussian16 team.

[42] Parraga H, Arranz F, Benito R, Borondo F. Above Saddle-Point Regions of Order in a Sea of Chaos in the Vibrational Dynamics of KCN. J Phys Chem A 2018;122:3433-3441. DOI: 10.1021/acs.jpca.8b00113.

[43] Robb M, Bernardi F, Olivucci M. Conical intersections as a mechanistic feature of organic photochemistry. Pure Appl Chem 1995;67:783-789. http://dx.doi.org/10.1351/pac199567050783.

[44] Domcke W, Yarkony D, Koppel H. Conical Intersections: Electronic Structure, Dynamics and Spectroscopy. World Scientific; Singapore: 2004.

[45] Polli D, Altoe P, Weingart O, Spillane K, Manzoni C, Brida D, Tomasello G, Orlandi G, Kukura P, Mathies R, Garavelli M, Cerullo G. Conical intersection dynamics of the primary photoisomerization event in vision. Nature 2010;467:440-443.

[46] Chen L, Gelin M, Chernyak V, Domcke W, Zhao Y. Dissipative dynamics at conical intersections: simulations with the hierarchy equations of motion method. Faraday Discussion 2016;194:61-80. DOI: 10.1039/C6FD00088F.

[47] Novotna P, Boon J, vand der Horst J, Pacakova V. Photoegradation of indigo in dichloromethane solution. Colouration Technology 2003;119:121-127. https://doi.org/10.1111/j.1478-4408.2003.tb00161.x. 